\title{
New Integral Inequalities via Generalized Preinvex Functions
}

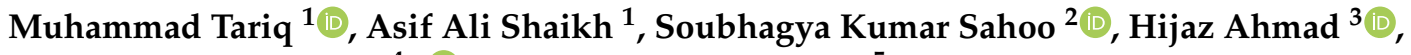 \\ Thanin Sitthiwirattham $4 * *$ (D) and Jiraporn Reunsumrit ${ }^{5}$ \\ 1 Department of Basic Sciences and Related Studies, Mehran University of Engineering and Technology, \\ Jamshoro 76062, Pakistan; captaintariq2187@gmail.com (M.T.); asif.shaikh@faculty.muet.edu.pk (A.A.S.) \\ 2 Department of Mathematics, Institute of Technical Education and Research, \\ Siksha 'O' Anusandhan University, Bhubaneswar 751030, India; soubhagyakumarsahoo@soa.ac.in \\ 3 Section of Mathematics, International Telematic University Uninettuno, Corso Vittorio Emanuele II, 39, \\ 00186 Roma, Italy; hijaz555@gmail.com \\ 4 Department of Mathematics, Faculty of Science and Technology, Suan Dusit University, \\ Bangkok 10300, Thailand \\ 5 Department of Mathematics, Faculty of Applied Science, King Mongkut's University of Technology North \\ Bangkok, Bangkok 10800, Thailand; jiraporn.r@sci.kmutnb.ac.th \\ * Correspondence: thanin_sit@dusit.ac.th
}

check for updates

Citation: Tariq, M.; Shaikh, A.A.; Sahoo, S.K.; Ahmad, H.; Sitthiwirattham, T.; Reunsumrit, J. New Integral Inequalities via Generalized Preinvex Functions. Axioms 2021, 10, 296. https:// doi.org/10.3390/axioms10040296

Academic Editor: Hans J. Haubold

Received: 29 September 2021

Accepted: 3 November 2021

Published: 7 November 2021

Publisher's Note: MDPI stays neutral with regard to jurisdictional claims in published maps and institutional affiliations.

Copyright: (c) 2021 by the authors. Licensee MDPI, Basel, Switzerland. This article is an open access article distributed under the terms and conditions of the Creative Commons Attribution (CC BY) license (https:/ / creativecommons.org/licenses/by/ $4.0 /)$.

\begin{abstract}
The theory of convexity plays an important role in various branches of science and engineering. The objective of this paper is to introduce a new notion of preinvex functions by unifying the $n$-polynomial preinvex function with the s-type $m$-preinvex function and to present inequalities of the Hermite-Hadamard type in the setting of the generalized $s$-type $m$-preinvex function. First, we give the definition and then investigate some of its algebraic properties and examples. We also present some refinements of the Hermite-Hadamard-type inequality using Hölder's integral inequality, the improved power-mean integral inequality, and the Hölder-İscan integral inequality. Finally, some results for special means are deduced. The results established in this paper can be considered as the generalization of many published results of inequalities and convexity theory.
\end{abstract}

Keywords: preinvex function; m-preinvex function; s-type convex function; Hölder's inequality; improved power-mean integral inequality

MSC: 26A51; 26A33; 26D07; 26D10; 26D15

\section{Introduction}

The theory of convex functions has become a rich source of inspiration in different fields of science. This hypothesis gives us some new refinements, which have been very fruitful in fostering mathematical strategies to tackle very complicated and difficult problems which emerge in physics, economics, engineering, and applied mathematics. Interested readers can refer to [1-4] for some classical convex functions and their related results.

The Hermite-Hadamard $(\mathrm{H}-\mathrm{H})$ inequality (see [5]) asserts that if a mapping $\psi: \mathbb{A} \subset$ $\mathbb{R} \rightarrow \mathbb{R}$ is convex in $\mathbb{A}$ for $\zeta, \varrho \in \mathbb{A}$, and $\varrho>\zeta$, then

$$
\psi\left(\frac{\varsigma+\varrho}{2}\right) \leq \frac{1}{\varrho-\varsigma} \int_{\varsigma}^{\varrho} \psi(x) d x \leq \frac{\psi(\varsigma)+\psi(\varrho)}{2}
$$

In the literature, it has been proven that the notion of the convex function has a very special relationship with the theory of inequalities. Among all the essential integral inequalities, the Hermite-Hadamard inequality holds a special place in the heart of all the mathematicians who work in the field of convexity theory. This inequality was first introduced by Hermite in 1881 in the journal Mathesis (see [6]). Until 1893, this inequality 
was nowhere mentioned nor did it exist in the literature of mathematics. In the year 1893, this inequality was proven by Hadamard (see [7]), and in the year 1974, Mitrinovic found Hermite's notes in Mathesis. Henceforth, this inequality was known as the HermiteHadamard inequality. The theory of inequalities has widespread views, robustness, and investigations in the variability of applied analysis. For some recent articles on inequalities, interested readers can see [8-20].

In the year 1981, Hanson [21] explored the idea of invexity as a generalization of differentiable convex functions. Later, Mond et al. [22] and Weir et al. [23] elaborated the concept of preinvexity, and they also explained how to apply this new concept in optimization problems.

The class of $m$-convex functions was introduced by G. Toader in [24]. After Toader, Latif [25] and Kalsoom [26] worked on the idea of $m$-preinvexity. In 2020, Toplu [27] introduced the $n$-polynomial convex function; explored its properties; and by applying this convexity, presented a novel version of the Hermite-Hadamard-type inequality.

Currently, due to the wide-spread applications of convex functions in different fields of pure and applied sciences, they have become a source of inspiration for many scientists. Numerous researchers have defined some new convex functions, and the idea of convexity has been stretched out in different directions including preinvexity, fractional calculus, quantum calculus, coordinates, interval valued calculus, fractal sets, etc. Motivated by ongoing research activities, in this article, we focus on introducing a new family of preinvex functions called the generalized $s$-type $m$-preinvex function. Furthermore, we have investigated its algebraic properties and examples to support the newly introduced concept. In addition, a novel version of the Hermite-Hadamard inequality and its refinements are presented to further enhance this new concept. Finally, some applications to special means are given as well. This is the main novelty of our article.

In this paper, we define and consider a new class of preinvex functions involving two arbitrary functions. We present some new Hermite-Hadamard-type integral inequalities. For proper choice of the arbitrary functions, we obtain few new and known notions for convex functions and preinvex functions as special cases. The results proved in this paper continue to hold for these special cases.

\section{Preliminaries}

In this section, we recall some known concepts related to preinvex functions, which we use extensively throughout the paper.

Let $\mathbb{A}$ be a nonempty closed set in $\mathbb{R}^{n}$. Let $\psi: \mathbb{A} \rightarrow \mathbb{R}$ be a continuous function, and let $\eta(.,):. \mathbb{A} \times \mathbb{A} \rightarrow \mathbb{R}^{n}$ be a continuous bifunction.

Definition 1 ([28]). A set $\mathbb{A}$ is said to be invex with respect to $\eta(.,$.$) if \varrho+\kappa \eta(\varsigma, \varrho) \in \mathbb{A}$, for the all mentioned conditions, i.e., for every $\varsigma, \varrho \in \mathbb{A}$ and $\kappa \in[0,1]$.

We might want to specify that Definition 1 of an invex set has a reasonable mathematical translation. This definition basically says that there is a path beginning from a point $\varrho$ which is contained in $\mathbb{A}$. We do not require that the point $\varsigma$ be one of the end points of the path. This perception assumes a significant part in our investigation. Note that if we prefer that $\zeta$ ought to be an end point of the path for each pair of points $\zeta, \varrho \in \mathbb{A}$, then at that point, $\eta(\varsigma, \varrho)=\varsigma-\varrho$; furthermore, subsequently, invexity becomes convexity. Accordingly, the facts confirm that each convex set is additionally an invex set with reference to $\eta(\varsigma, \varrho)=\varsigma-\varrho$; yet, the converse is not really obvious in general (see $[28,29])$.

Note that in the literature, invex set $\mathbb{A}$ is also known as a $\eta$-connected set.

Definition 2 ([22]). Let $\mathbb{A} \neq \varnothing \in \mathbb{R}$ be an invex set with respect to $\eta: \mathbb{A} \times \mathbb{A} \neq \varnothing \rightarrow \mathbb{R}$. Then, the function $\psi: \mathbb{A} \rightarrow \mathbb{R}$ is said to be preinvex with respect to $\eta$ if

$$
\psi(\varrho+\kappa \eta(\varsigma, \varrho)) \leq \kappa \psi(\varsigma)+(1-\kappa) \psi(\varrho), \quad \forall \varsigma, \varrho \in \mathbb{A}, \kappa \in[0,1] .
$$


The function $-\psi$ is said to be preincave if and only if $\psi$ is preinvex.

In the year 2007, Noor [30] applied the concept of preinvexity to establish the HermiteHadamard-type inequality, which is stated as follows:

Theorem 1. Let $\psi: \mathbb{A}=[\varsigma, \varsigma+\eta(\varrho, \varsigma)] \rightarrow(0, \infty)$ be a preinvex function on the interval of real numbers $\mathbb{A}^{\circ}$ and $\varsigma, \varrho \in \mathbb{A}$ with $\varsigma<\varsigma+\eta(\varrho, \varsigma)$. Then, the following Hermite-Hadamard-type inequalities for the preinvex function hold:

$$
\psi\left(\frac{2 \varsigma+\eta(\varrho, \varsigma)}{2}\right) \leq \frac{1}{\eta(\varrho, \varsigma)} \int_{\varsigma}^{\varsigma+\eta(\varrho, \varsigma)} \psi(x) d x \leq \frac{\psi(\varsigma)+\psi(\varrho)}{2} .
$$

In 2011, a group of mathematicians, i.e., A. Barani, A.G. Gahazanfari, and S.S. Dragomir, worked on the idea of preinvexity in [31] and established the Hermite-Hadamard type for the differentiable preinvex function, which is stated as follows:

Theorem 2. Let $\mathbb{A} \subseteq \mathbb{R}$ be an open invex subset with respect to $\eta: \mathbb{A} \times \mathbb{A} \rightarrow \mathbb{R}$. Suppose $\psi: \mathbb{A} \rightarrow \mathbb{R}$ is a differentiable function. If $\left|\psi^{\prime}\right|$ is preinvex on $\mathbb{A}$, then for every $\varsigma, \varrho \in \mathbb{A}$ with $\eta(\varrho, \varsigma) \neq 0$. Then, the following Hermite-Hadamard-type inequalities for preinvex hold:

$$
\left|\frac{\psi(\varsigma)+\psi(\varsigma+\eta(\varrho, \varsigma)}{2}-\frac{1}{\eta(\varrho, \varsigma)} \int_{\varsigma}^{\varsigma+\eta(\varrho, \varsigma)} \psi(x) d x\right| \leq \frac{|\eta(\varrho, \varsigma)|}{8}[\psi(\varsigma)+\psi(\varrho)] .
$$

Soon after these results, different authors presented and incorporated their perspectives into the idea of preinvex functions. Interested readers are encouraged to go through [32-39]. To proceed with our main results, we need the following hypothesis regarding the bifunction $\eta$, which is due to Mohan and Neogy [40].

\section{Condition-C:}

Let $\mathbb{A} \subset R^{n}$ be an open invex subset with respect to $\eta: \mathbb{A} \times \mathbb{A} \rightarrow \mathbb{R}$. For any $\varsigma, \varrho \in \mathbb{A}$, and $\kappa \in[0,1]$,

$$
\begin{array}{r}
\eta(\varrho, \varrho+\kappa \eta(\varsigma, \varrho))=-\kappa \eta(\varsigma, \varrho) \\
\eta(\varsigma, \varrho+\kappa \eta(\varsigma, \varrho))=(1-\kappa) \eta(\varsigma, \varrho) .
\end{array}
$$

For any $\varsigma, \varrho \in \mathbb{A}$, and $\kappa_{1}, \kappa_{2} \in[0,1]$, from Condition $C$, we have

$$
\eta\left(\varrho+\kappa_{2} \eta(\varsigma, \varrho), \varrho+\kappa_{1} \eta(\varsigma, \varrho)\right)=\left(\kappa_{2}-\kappa_{1}\right) \eta(\varsigma, \varrho) .
$$

\section{Extended Condition-C:}

Let $\mathbb{A} \subset R^{n}$ be an open invex subset with respect to $\eta: \mathbb{A} \times \mathbb{A} \rightarrow \mathbb{R}$. For any $\varsigma, \varrho \in \mathbb{A}$, and $\kappa \in[0,1]$,

$$
\begin{gathered}
\eta(\varrho+\kappa \eta(\varsigma, \varrho), \varrho+(1-\kappa) \eta(\varsigma, \varrho))=(\kappa-1+\kappa) \eta(\varsigma, \varrho) \\
\eta(\varrho+\kappa \eta(\varsigma, \varrho), \varrho+(1-\kappa) \eta(\varsigma, \varrho))=(2 \kappa-1) \eta(\varsigma, \varrho) .
\end{gathered}
$$

In the last few decades, a large of number of mathematicians have worked and polished the idea of preinvexity in different directions. Recently, A. Latif [25] introduced and explored generalized $m$-preinvexity, which is given as follows:

Definition 3. Let $\psi: \mathbb{A} \rightarrow \mathbb{R}$ be a function on the invex set $\mathbb{A} \subseteq\left[0, b^{*}\right], b^{*}>0$; then, $\psi$ is said to be an $m$-preinvex with respect to $\eta$ for some fixed $m \in(0,1]$ if

$$
\psi(\varrho+\kappa \eta(\varsigma, \varrho)) \leq(1-\kappa) \psi(\varrho)+m \kappa \psi\left(\frac{\varsigma}{m}\right),
$$


holds for every $\varsigma, \varrho \in \mathbb{A}, \kappa \in[0,1]$.

Definition 4 ([41]). A non-negative function $\psi: \mathbb{A} \rightarrow \mathbb{R}$ is called an s-type convex function if for every $\varsigma, \varrho \in \mathbb{A}, s \in[0,1]$, and $\kappa \in[0,1]$, the following inequality holds:

$$
\psi(\kappa \varsigma+(1-\kappa) \varrho) \leq[1-(s(1-\kappa))] \psi(\varsigma)+[1-s \kappa] \psi(\varrho) .
$$

Definition 5 ([27]). A non-negative real-valued function $\psi: \mathbb{A} \rightarrow \mathbb{R}$ is called an n-polynomial convex function if

$$
\psi(\kappa \varsigma+(1-\kappa) \varrho) \leq \frac{1}{n} \sum_{i=1}^{n}\left[1-(1-\kappa)^{i}\right] \psi(\varsigma)+\frac{1}{n} \sum_{i=1}^{n}\left[1-\kappa^{i}\right] \psi(\varrho),
$$

holds for every $\varsigma, \varrho \in \mathbb{A}, \kappa \in[0,1], s \in[0,1]$, and $n \in \mathbb{N}$.

Definition 6 ([2]). An inequality of the form

$$
(\psi(\varsigma)-\psi(\varrho))(\varphi(\varsigma)-\varphi(\varrho)) \geq 0, \quad \forall \varsigma, \varrho \in \mathbb{R} .
$$

is said to be similarly ordered.

By ongoing research activities and owing to the recent trend in preinvexity, we organize the article as follows. In Section 3, we will define and explore the newly introduced idea about generalized s-type $m$-preinvex functions and its algebraic properties. In Section 4 , we present a novel version of the Hermite-Hadamard-type inequality using the new notion of preinvexity. In Section 5, employing a published lemma, we present some new refinements of the Hermite-Hadamard-type inequality. All results presented in this paper are true and new to the literature.

\section{Generalized Preinvexity and Its Properties}

In this section, we are going to introduce a new notion of the preinvex function, namely the generalized $s$-type $m$-preinvex function, and study some of its related algebraic properties.

Definition 7. Let $\mathbb{A} \subset \mathbb{R}$ be a nonempty m-invex set with respect to $\eta: \mathbb{A} \times \mathbb{A} \times(0,1] \rightarrow \mathbb{R}$. Then, $\psi: \mathbb{A} \rightarrow \mathbb{R}$ is said to be a generalized s-type m-preinvex if

$$
\psi(\varrho+\kappa \eta(\varsigma, \varrho)) \leq \frac{1}{n} \sum_{i=1}^{n}\left(1-(s \kappa)^{i}\right) \psi(\varrho)+\frac{1}{n} \sum_{i=1}^{n}\left(1-(s(1-\kappa))^{i}\right) m^{i} \psi\left(\frac{\varsigma}{m^{i}}\right)
$$

holds for every $\varsigma, \varrho \in \mathbb{A}, s \in[0,1], n \in \mathbb{N}, m \in(0,1]$, and $\kappa \in[0,1]$.

\section{Remark 1.}

(i) If we choose $n=1$ in Definition 7, then we have a new definition of an s-type m-preinvex function:

$$
\psi(m \varrho+\kappa \eta(\varsigma, \varrho, m)) \leq(1-s \kappa) \psi(\varrho)+(1-(s(1-\kappa))) \psi(\varsigma) .
$$

(ii) Taking $n=s=m=1$ in Definition 7, we have the definition of a preinvex function given by Weir and Mond [22].

(iii) Taking $n=m=1$ and $\eta(\varsigma, \varrho)=\varsigma-\varrho$ in Definition 7 , we have the definition of an s-type convex function given by İscan et al. [41].

(iv) Taking $n=s=m=1$ and $\eta(\varsigma, \varrho)=\varsigma-\varrho$ in Definition 7, we have the definition of $a$ convex function which is investigated by Niculescu et al. [2]. 
(v) If $n=2$, then we obtain the following new inequality for a 2-polynomial s-type m-preinvex function:

$$
\begin{aligned}
& \psi(\varrho+\kappa \eta(\varsigma, \varrho)) \\
& \quad \leq \frac{1}{2}\left[\left(2-s \kappa-s^{2} \kappa^{2}\right) \psi(\varrho)+(1-(s(1-\kappa))) m \psi\left(\frac{\varsigma}{m}\right)+\left(1-(s(1-\kappa))^{2}\right) m^{2} \psi\left(\frac{\varsigma}{m^{2}}\right)\right] .
\end{aligned}
$$

Lemma 1. The following inequalities

$$
m \kappa \leq \frac{1}{n} \sum_{i=1}^{n} m^{i}\left(1-(s(1-\kappa))^{i}\right) \text { and }(1-\kappa) \leq \frac{1}{n} \sum_{i=1}^{n}\left(1-(s \kappa)^{i}\right)
$$

hold for all $\kappa \in[0,1], m \in(0,1], n \in \mathbb{N}$, and $s \in[0,1]$.

Proof. First, we will prove that the inequality $\forall \kappa \in[0,1]$ and $n \in N$ :

$$
m \kappa \leq \frac{1}{n} \sum_{i=1}^{n} m^{i}\left(1-(s(1-\kappa))^{i}\right)
$$

The following inequality is known as Bernoulli's inequality in mathematical analysis:

$$
(1-m \kappa)^{n} \geq 1-m n \kappa, \quad \forall \kappa \in[0,1] \text { and } n \in N \text {. }
$$

From the above inequality, we obtain

$$
\begin{gathered}
\frac{1}{n} \sum_{i=1}^{n}(1-m \kappa)^{i-1}=\frac{1-(1-m \kappa)^{n}}{m n \kappa} \leq 1 . \\
n(1-m \kappa)\left[-1+\frac{1}{n} \sum_{i=1}^{n}(1-m \kappa)^{i-1}\right]=-n(1-m \kappa)+\sum_{i=1}^{n}(1-m \kappa)^{i} \leq 0,
\end{gathered}
$$

and then we have

$$
m \kappa \leq \frac{1}{n} \sum_{i=1}^{n} m^{i}\left(1-(s(1-\kappa))^{i}\right)
$$

The interested reader can also prove the inequality $(1-\kappa) \leq \frac{1}{n} \sum_{i=1}^{n}\left(1-(s \kappa)^{i}\right)$ using the same procedure as above.

Lemma 2. The following inequalities

$$
m(1-(s(1-\kappa))) \leq \frac{1}{n} \sum_{i=1}^{n} m^{i}\left(1-(s(1-\kappa))^{i}\right) \quad \text { and } \quad(1-s \kappa) \leq \frac{1}{n} \sum_{i=1}^{n}\left(1-(s \kappa)^{i}\right)
$$

hold for all $\kappa \in[0,1], m \in(0,1], n \in \mathbb{N}$, and $s \in[0,1]$.

Proof. The rest of the proof is clearly seen.

Proposition 1. Every non-negative $m$-preinvex function is a generalized s-type m-preinvex function for $s \in[0,1], m \in(0,1], n \in \mathbb{N}$, and $\kappa \in[0,1]$. 
Proof. By using Lemma 1 and the definition of $m$-preinvexity for $s \in[0,1], m \in(0,1]$, and $\kappa \in[0,1]$, we have

$$
\begin{aligned}
& \psi(\varrho+\kappa \eta(\varsigma, \varrho)) \leq(1-\kappa) \psi(\varrho)+m \kappa \psi\left(\frac{\varsigma}{m}\right) \\
& \leq \frac{1}{n} \sum_{i=1}^{n}\left(1-(s \kappa)^{i}\right) \psi(\varrho)+\frac{1}{n} \sum_{i=1}^{n}\left(1-(s(1-\kappa))^{i}\right) m^{i} \psi\left(\frac{\varsigma}{m^{i}}\right) .
\end{aligned}
$$

Proposition 2. Every non-negative s-type m-preinvex function is a generalized s-type m-preinvex function for $s \in[0,1], m \in(0,1], n \in \mathbb{N}$, and $\kappa \in[0,1]$.

Proof. By using Lemma 2 and the definition of $s$-type $m$-preinvexity for $s \in[0,1], m \in$ $(0,1]$, and $\kappa \in[0,1]$, we have

$$
\begin{aligned}
& \psi(\varrho+\kappa \eta(\varsigma, \varrho)) \leq(1-s \kappa) \psi(\varrho)+(1-(s(1-\kappa))) m^{i} \psi\left(\frac{\varsigma}{m^{i}}\right), \\
& \leq \frac{1}{n} \sum_{i=1}^{n}\left(1-(s \kappa)^{i}\right) \psi(\varrho)+\frac{1}{n} \sum_{i=1}^{n}\left(1-(s(1-\kappa))^{i}\right) m^{i} \psi\left(\frac{\varsigma}{m^{i}}\right) .
\end{aligned}
$$

Proposition 3. Every non-negative generalized s-type m-preinvex function for $s \in[0,1], m \in(0,1]$, $n \in \mathbb{N}$, and $\kappa \in[0,1]$ is an $(h, m)$-preinvex function with $h(\kappa)=\frac{1}{n} \sum_{i=1}^{n}\left(1-(s(1-\kappa))^{i}\right)$.

Proof. Using the definition of generalized $s$-type $m$-preinvexity for $s \in[0,1], m \in(0,1]$, $\kappa \in[0,1]$, and the condition mentioned for $h(\kappa)=\frac{1}{n} \sum_{i=1}^{n}\left(1-(s(1-\kappa))^{i}\right)$, we have

$$
\begin{gathered}
\psi(\varrho+\kappa \eta(\varsigma, \varrho)) \leq \frac{1}{n} \sum_{i=1}^{n}\left(1-(s \kappa)^{i}\right) \psi(\varrho)+\frac{1}{n} \sum_{i=1}^{n}\left(1-(s(1-\kappa))^{i}\right) m^{i} \psi\left(\frac{\varsigma}{m^{i}}\right), \\
\leq h(1-\kappa) \psi(\varrho)+m h(\kappa) \psi\left(\frac{\varsigma}{m}\right) .
\end{gathered}
$$

The beauty of the new class of generalized $s$-type $m$-preinvexity is that it is larger in comparison to some known classes of convex and preinvex functions.

Now, utilising the above-mentioned propositions, we present some examples in the manner of the newly defined concept.

Example 1. Since $\psi(x)=e^{x}, \quad \forall x \geq 0$ is a non-negative convex function, it is a non-negative preinvex function for $m=1$, because every convex function is a preinvex function (see [42]). By using Proposition 1, it is a generalized s-type m-preinvex function.

Note: Every convex function is a preinvex, but the converse is not true. For example, $\psi(x)=-|x|$.

Thus, we are now able to present an example of a generalized $s$-type $m$-preinvex function with respect to $\eta$ on a set $\mathbb{X}$, but it is a non-negative and not convex function.

Example 2. Let $\psi(x): \mathbb{R}^{+} \rightarrow \mathbb{R}^{+}$be a function, which is defined by

$\psi(x)= \begin{cases}x & ; 0 \leq x \leq 1 \\ 1 & ; x>1\end{cases}$

and 


$$
\eta(\varsigma, \varrho)= \begin{cases}\varsigma-\varrho, & ; \varsigma \leq 0, \varrho \leq 0 \\ \varsigma-\varrho ; 0 \leq \varsigma \leq 1, \varrho \leq 1 \\ -2-\varrho \quad ; \varsigma \leq 0,0 \leq \varrho \leq 1 \\ 2-\varrho \quad ; 0 \leq \varsigma \leq 1, \varrho \leq 0 .\end{cases}
$$

If $m=1$, then the above non-negative function $\psi(x)$ is an m-preinvex function but not convex. According to Proposition 1, every non-negative m-preinvex function is a generalized s-type $m$-preinvex function with respect to $\eta$ on $X$ if $m=1$.

Example 3. Let $\psi(x): \mathbb{R}^{+} \rightarrow \mathbb{R}^{+}$be a function, which is defined by

$\psi(x)=\left\{\begin{array}{l}x+1 \quad ; 0 \leq x \leq 1 \\ 1 \quad ; x>1\end{array}\right.$

and

$\eta(\varsigma, \varrho)=\left\{\begin{array}{l}\varsigma+\varrho ; \varsigma \leq \varrho \\ 2(\varsigma+\varrho) ; \varsigma>\varrho\end{array}\right.$

$\forall \varsigma, \varrho \in \mathbb{R}^{+}=[0,+\infty)$. Using the same logic as above, it is a generalized s-type preinvex function with respect to $\eta$ on $X$.

Next, we explore some algebraic properties of the newly introduced concept.

Theorem 3. Let $\psi, \varphi: \mathbb{X}=[\varsigma, \varrho] \rightarrow \mathbb{R}$ be two generalized s-type m-preinvex functions; then,

(i) The sum of the above functions is a generalized s-type m-preinvex function.

(ii) The scalar multiplication of the above function is a generalized s-type m-preinvex function.

\section{Proof.}

(i) For all $\varsigma, \varrho \in \mathbb{X}, s \in[0,1], m \in(0,1]$, and $\kappa \in[0,1]$, we have

$$
\begin{aligned}
& (\psi+\varphi)(\varrho+\kappa \eta(\varsigma, \varrho)) \\
& =\psi(\varrho+\kappa \eta(\varsigma, \varrho))+\varphi(\varrho+\kappa \eta(\varsigma, \varrho)) \\
& \leq \frac{1}{n} \sum_{i=1}^{n}\left(1-(s \kappa)^{i}\right) \psi(\varrho)+\frac{1}{n} \sum_{i=1}^{n}\left(1-(s(1-\kappa))^{i}\right) m^{i} \psi\left(\frac{\varsigma}{m^{i}}\right) \\
& +\frac{1}{n} \sum_{i=1}^{n}\left(1-(s \kappa)^{i}\right) \varphi(\varrho)+\frac{1}{n} \sum_{i=1}^{n}\left(1-(s(1-\kappa))^{i}\right) m^{i} \varphi\left(\frac{\varsigma}{m^{i}}\right) \\
& =\frac{1}{n} \sum_{i=1}^{n}\left(1-(s \kappa)^{i}\right)[\psi(\varrho)+\varphi(\varrho)]+\frac{1}{n} \sum_{i=1}^{n}\left(1-(s(1-\kappa))^{i}\right)\left[m^{i} \psi\left(\frac{\varsigma}{m^{i}}\right)+m^{i} \varphi\left(\frac{\varsigma}{m^{i}}\right)\right] \\
& =\frac{1}{n} \sum_{i=1}^{n}\left(1-(s \kappa)^{i}\right)(\psi+\varphi)(\varrho)+\frac{1}{n} \sum_{i=1}^{n}\left(1-(s(1-\kappa))^{i}\right) m^{i}(\psi+\varphi)\left(\frac{\varsigma}{m^{i}}\right) .
\end{aligned}
$$

(ii) For all $\varsigma, \varrho \in \mathbb{X}, s \in[0,1], c \in \mathbb{R}(c \geq 0), m \in(0,1]$, and $\kappa \in[0,1]$, we have

$$
\begin{aligned}
& (c \psi)(\varrho+\kappa \eta(\varsigma, \varrho)) \\
& \leq c\left[\frac{1}{n} \sum_{i=1}^{n}\left(1-(s \kappa)^{i}\right) \psi(\varrho)+\frac{1}{n} \sum_{i=1}^{n}\left(1-(s(1-\kappa))^{i}\right) m^{i} \psi\left(\frac{\varsigma}{m^{i}}\right)\right] \\
& =\frac{1}{n} \sum_{i=1}^{n}\left(1-(s \kappa)^{i}\right) c \psi(\varrho)+\frac{1}{n} \sum_{i=1}^{n}\left(1-(s(1-\kappa))^{i}\right) c m^{i} \psi\left(\frac{\varsigma}{m^{i}}\right) \\
& =\frac{1}{n} \sum_{i=1}^{n}\left(1-(s \kappa)^{i}\right)(c \psi)(\varrho)+\frac{1}{n} \sum_{i=1}^{n}\left(1-(s(1-\kappa))^{i}\right) m^{i}(c \psi)\left(\frac{\varsigma}{m^{i}}\right) .
\end{aligned}
$$

This completes the proof. 


\section{Remark 2.}

(i) Choosing $n=1$ in Theorem 3, it is easily seen that $\psi+\varphi$ and $c \psi$ are s-type $m$-preinvex functions.

(ii) Choosing $s=m=1$ in Theorem 3, it is easily seen that $\psi+\varphi$ and $c \psi$ are generalized preinvex functions.

(iii) Choosing $n=s=m=1$ in Theorem 3, it is easily seen that $\psi+\varphi$ and $c \psi$ are preinvex functions.

(iv) Choosing $n=m=1$ and $\eta(\varsigma, \varrho, m)=\varsigma-m \varrho$ in Theorem 3, it is easily seen that $\psi+\varphi$ and $c \psi$ are s-type convex functions.

(v) Choosing $\eta(\varsigma, \varrho)=\varsigma-\varrho$ and $n=s=m=1$ in Theorem 3, it is easily seen that $\psi+\varphi$ and $c \psi$ are convex functions.

Theorem 4. Let $\psi: \mathbb{X} \rightarrow \mathbb{Y}$ be a generalized s-type $m$-preinvex and $\varphi: \mathbb{Y} \rightarrow \mathbb{R}$ be a nondecreasing function. Then the composition of these functions is a generalized s-type m-preinvex for $s \in[0,1], m \in(0,1]$, and $\kappa \in[0,1]$.

Proof. For all $\varsigma, \varrho \in \mathbb{X}, s \in[0,1], m \in(0,1]$, and $\kappa \in[0,1]$, we have

$$
\begin{aligned}
& (\varphi \circ \psi)(\varrho+\kappa \eta(\varsigma, \varrho)) \\
& =\varphi(\psi(\varrho+\kappa \eta(\varsigma, \varrho))) \\
& \leq \varphi\left[\frac{1}{n} \sum_{i=1}^{n}\left(1-(s \kappa)^{i}\right) \psi(\varrho)+\frac{1}{n} \sum_{i=1}^{n}\left(1-(s(1-\kappa))^{i}\right) m^{i} \psi\left(\frac{\varsigma}{m^{i}}\right)\right] \\
& \leq \frac{1}{n} \sum_{i=1}^{n}\left(1-(s \kappa)^{i}\right) \varphi(\psi(\varrho))+\frac{1}{n} \sum_{i=1}^{n}\left(1-(s(1-\kappa))^{i}\right) m^{i} \varphi\left(m^{i} \psi\left(\frac{\varsigma}{m^{i}}\right)\right) \\
& =\frac{1}{n} \sum_{i=1}^{n}\left(1-(s \kappa)^{i}\right)(\varphi \circ \psi)(\varrho)+\frac{1}{n} \sum_{i=1}^{n}\left(1-(s(1-\kappa))^{i}\right) m^{2 i}(\varphi \circ \psi)\left(\frac{\varsigma}{m^{i}}\right) .
\end{aligned}
$$

\section{Remark 3.}

(i) Choosing $n=s=1$ in Theorem 4, then

$$
(\varphi \circ \psi)(m \varrho+\kappa \eta(\varsigma, \varrho, m)) \leq(1-\kappa)(\varphi \circ \psi)(\varrho)+\kappa m^{2}(\varphi \circ \psi)\left(\frac{\varsigma}{m}\right) .
$$

(ii) Choosing $n=s=m=1$ in Theorem 4 , then

$$
(\varphi \circ \psi)(\varrho+\kappa \eta(\varsigma, \varrho)) \leq(1-\kappa)(\varphi \circ \psi)(\varrho)+\kappa(\varphi \circ \psi)(\varsigma)
$$

(iii) If we put $n=m=1$ and $\eta(\varsigma, \varrho)=\varsigma-\varrho$ in Theorem 4 , then

$$
(\varphi \circ \psi)(\kappa \varsigma+(1-\kappa) \varrho) \leq[1-s \kappa](\varphi \circ \psi)(\varrho)+[1-(s(1-\kappa))](\varphi \circ \psi)(\varsigma) .
$$

Theorem 5. Let $0<\varsigma<\varrho, \psi_{j}: \mathbb{A}=[\varsigma, \varrho] \rightarrow[0,+\infty)$ be a class of generalized s-type $m-$ preinvex functions, and $\psi(u)=\sup _{j} \psi_{j}(u)$. Then, $\psi$ is a generalized s-type m-preinvex for $s \in[0,1], m \in(0,1]$, and $\kappa \in[0,1]$, and $U=\left\{\tau \in[\varsigma, \varrho]: \psi\left(\tau_{i}\right)<\infty\right\}$ is an interval. 
Proof. Let $\varsigma, \varrho \in U, s \in[0,1], m \in(0,1]$, and $\kappa \in[0,1]$; then,

$$
\begin{aligned}
& \psi(\varrho+\kappa \eta(\varsigma, \varrho)) \\
& =\sup _{j} \psi_{j}(\varrho+\kappa \eta(\varsigma, \varrho)) \\
& \leq \frac{1}{n} \sum_{i=1}^{n}\left(1-(s \kappa)^{i}\right) \sup _{j} \psi_{j}(\varrho)+\frac{1}{n} \sum_{i=1}^{n}\left(1-(s(1-\kappa))^{i}\right) m^{i} \sup _{j} \psi_{j}\left(\frac{\varsigma}{m^{i}}\right) \\
& =\frac{1}{n} \sum_{i=1}^{n}\left(1-(s \kappa)^{i}\right) \psi(\varrho)+\frac{1}{n} \sum_{i=1}^{n}\left(1-(s(1-\kappa))^{i}\right) m^{i} \psi\left(\frac{\varsigma}{m^{i}}\right)<\infty .
\end{aligned}
$$

This is the required proof.

Theorem 6. If $\psi_{i}: \mathbb{R}^{n} \rightarrow \mathbb{R}$ is a generalized s-type m-preinvex function with respect to $\eta$ for $s \in[0,1], m \in(0,1]$, and $\kappa \in[0,1]$, then the set $\mathbb{M}=\left\{\tau \in \mathbb{R}: \psi_{i}(\tau) \leq 0, i=1,2,3, \ldots, n\right\}$ is an $m$-invex set.

Proof. Since $\psi_{i}(\tau),(i=1,2,3, \ldots, n)$ are generalized $s$-type $m$-preinvex functions with respect to $\eta$ for $s \in[0,1], m \in(0,1]$, and $\kappa \in[0,1]$, then for all $\varsigma, \varrho \in \mathbb{R}^{n}$,

$$
\psi_{i}(\varrho+\kappa \eta(\varsigma, \varrho)) \leq \frac{1}{n} \sum_{i=1}^{n}\left(1-(s \kappa)^{i}\right) \psi(\varrho)+\frac{1}{n} \sum_{i=1}^{n}\left(1-(s(1-\kappa))^{i}\right) m^{i} \psi\left(\frac{\varsigma}{m^{i}}\right),
$$

holds, where $i=1,2,3, \ldots, n$

When $\varsigma, \varrho \in \mathbb{M}$, we know $\psi_{i}(\varsigma) \leq 0$ and $\psi_{i}(\varrho) \leq 0$; from the above inequality, it yields that

$$
\psi_{i}(\varrho+\kappa \eta(\varsigma, \varrho)) \leq 0, \quad i=1,2,3, \ldots, n .
$$

That is, $\varrho+\kappa \eta(\varsigma, \varrho) \in \mathbb{M}$. Hence, $\mathbb{M}$ is an $m$-invex set.

Theorem 7. If $\psi: \mathbb{X} \subseteq \mathbb{R}^{n} \rightarrow \mathbb{R}$ is a generalized s-type m-preinvex function on $m$-invex set $\mathbb{X}$ with respect to $\eta$ for $s \in[0,1], m \in(0,1]$, and $\kappa \in[0,1]$, then the function $\psi$ is also a generalized quasi $m$-preinvex function on $m$-invex set $\mathbb{X}$ with respect to $\eta$.

Proof. Since $\psi$ is a generalized $s$-type $m$-preinvex function with respect to $\eta$ for $s \in[0,1]$, $m \in(0,1]$, and $\kappa \in[0,1]$, and we assume that $\frac{1}{n} \sum_{i=1}^{n} m^{i} \psi\left(\frac{\varsigma}{m^{i}}\right) \leq \psi(\varrho)$, then for all $\varsigma, \varrho \in \mathbb{X}$, we have

$$
\begin{aligned}
& \psi(\varrho+\kappa \eta(\varsigma, \varrho)) \\
& \leq \frac{1}{n} \sum_{i=1}^{n}\left(1-(s \kappa)^{i}\right) \psi(\varrho)+\frac{1}{n} \sum_{i=1}^{n}\left(1-(s(1-\kappa))^{i}\right) m^{i} \psi\left(\frac{\varsigma}{m^{i}}\right) \\
& \leq\left[\frac{1}{n} \sum_{i=1}^{n}\left(1-(s(1-\kappa))^{i}\right)+\frac{1}{n} \sum_{i=1}^{n}\left(1-(s \kappa)^{i}\right)\right] \psi(\varrho) \\
& \leq \psi(\varrho) .
\end{aligned}
$$

In the same manner, let $\psi(\varrho) \leq \frac{1}{n} \sum_{i=1}^{n} m^{i} \psi\left(\frac{\varsigma}{m^{i}}\right)$; for all $\varsigma, \varrho \in \mathbb{X}$, we can also obtain

$$
\psi(\varrho+\kappa \eta(\varsigma, \varrho)) \leq \frac{1}{n} \sum_{i=1}^{n} m^{i} \psi\left(\frac{\varsigma}{m^{i}}\right)
$$

Consequently,

$$
\psi(\varrho+\kappa \eta(\varsigma, \varrho)) \leq \max \{\psi(\varsigma), \psi(\varrho)\}
$$


That is, $\psi: \mathbb{X} \subseteq \mathbb{R}^{n} \rightarrow \mathbb{R}$ is a generalized quasi $m$-preinvex function on $m$-invex set $\mathbb{X}$ with respect to $\eta$.

Theorem 8. If $\psi: \mathbb{R}_{\circ} \rightarrow \mathbb{R}_{\circ}$ is a generalized s-type m-preinvex function with respect to $\eta: \mathbb{R}_{\circ} \times$ $\mathbb{R}_{\circ} \times(0,1] \rightarrow \mathbb{R}_{\circ}$ for $s \in[0,1], m \in(0,1]$, and $\kappa \in[0,1]$. Assume that $\psi$ is monotone decreasing and $\eta$ is monotone increasing regarding $m$ for fixed $\varsigma, \varrho \in \mathbb{R}_{\circ}$ and $m_{1} \leq m_{2} \quad\left(m_{1}, m_{2} \in(0,1]\right)$. If $\psi$ is a generalized s-type $m_{1}$-preinvex function on $\mathbb{R}_{\circ}$ with respect to $\eta$, then $\psi$ is a generalized s-type $m_{2}$-preinvex function on $\mathbb{R}_{\circ}$ with respect to $\eta$.

Proof. Since $\psi$ is a generalized $s$-type $m_{1}$-preinvex function, for all $\varsigma, \varrho \in \mathbb{R}_{\mathrm{o}}$,

$$
\psi(\varrho+\kappa \eta(\varsigma, \varrho)) \leq \frac{1}{n} \sum_{i=1}^{n}\left(1-(s \kappa)^{i}\right) \psi(\varrho)+\frac{1}{n} \sum_{i=1}^{n}\left(1-(s(1-\kappa))^{i}\right) m_{1}^{i} \psi\left(\frac{\varsigma}{m_{1}^{i}}\right) .
$$

Combining the monotone decreasing of the function $\psi$ with the monotone increasing of the mapping $\eta$ regarding $m$ for fixed $\varsigma, \varrho \in \mathbb{R}_{\circ}$ and $\frac{1}{n} \sum_{i=1}^{n} m_{1}^{i} \leq \frac{1}{n} \sum_{i=1}^{n} m_{2}^{i}$, it follows that

$$
\begin{aligned}
& \frac{1}{n} \sum_{i=1}^{n}\left(1-(s \kappa)^{i}\right) \psi(\varrho)+\frac{1}{n} \sum_{i=1}^{n}\left(1-(s(1-\kappa))^{i}\right) m_{1}^{1} \psi\left(\frac{\varsigma}{m_{1}^{i}}\right) \\
& \leq \frac{1}{n} \sum_{i=1}^{n}\left(1-(s \kappa)^{i}\right) \psi(\varrho)+\frac{1}{n} \sum_{i=1}^{n}\left(1-(s(1-\kappa))^{i}\right) m_{2}^{i} \psi\left(\frac{\varsigma}{m_{2}^{i}}\right) .
\end{aligned}
$$

Finally, we have

$$
\psi(\varrho+\kappa \eta(\varsigma, \varrho)) \leq \frac{1}{n} \sum_{i=1}^{n}\left(1-(s \kappa)^{i}\right) \psi(\varrho)+\frac{1}{n} \sum_{i=1}^{n}\left(1-(s(1-\kappa))^{i}\right) m_{2}^{i} \psi\left(\frac{\varsigma}{m_{2}^{i}}\right)
$$

Hence, $\psi$ is a generalized $s$-type $m_{2}$-preinvex function on $\mathbb{R}_{\circ}$ with respect to $\eta$ for fixed $s \in[0,1]$ and $m \in[0,1]$, which ends the proof.

Theorem 9. Let $\psi, \varphi: \mathbb{A}=[\varsigma, \varrho] \rightarrow \mathbb{R}$ be two generalized s-type m-preinvex and similarly ordered functions and $[1-(s(1-\kappa))]+[1-s \kappa] \leq 1$; then, the product $\psi \varphi$ is a generalized s-type $m$-preinvex function with respect to $\eta$ for $s \in[0,1], m \in(0,1]$, and $\kappa \in[0,1]$.

Proof. Let $\psi, \varphi$ be a generalized s-type $m$-preinvex function with respect to the same $\eta$, $s \in[0,1], m \in(0,1]$, and $\kappa \in[0,1]$; then,

$$
\begin{aligned}
& \psi(\varrho+\kappa \eta(\varsigma, \varrho)) \varphi(\varrho+\kappa \eta(\varsigma, \varrho)) \\
& \leq\left[\frac{1}{n} \sum_{i=1}^{n}\left(1-(s \kappa)^{i}\right) \psi(\varrho)+\frac{1}{n} \sum_{i=1}^{n}\left(1-(s(1-\kappa))^{i}\right) m^{i} \psi\left(\frac{\varsigma}{m^{i}}\right)\right] \\
& \times\left[\frac{1}{n} \sum_{i=1}^{n}\left(1-(s \kappa)^{i}\right) \varphi(\varrho)+\frac{1}{n} \sum_{i=1}^{n}\left(1-(s(1-\kappa))^{i}\right) m^{i} \varphi\left(\frac{\varsigma}{m^{i}}\right)\right] \\
& \leq \frac{1}{n^{2}} \sum_{i=1}^{n}(1-s \kappa)^{2 i} \psi(\varrho) \varphi(\varrho)+\frac{1}{n^{2}} \sum_{i=1}^{n}\left(1-(s(1-\kappa))^{i}\right)^{2} m^{2 i} \psi\left(\frac{\varsigma}{m^{i}}\right) \varphi\left(\frac{\varsigma}{m^{i}}\right) \\
& +\frac{1}{n^{2}} \sum_{i=1}^{n}\left(1-(s(1-\kappa))^{i}\right)\left(1-(s \kappa)^{i}\right)\left[m^{i} \psi\left(\frac{\varsigma}{m^{i}}\right) \varphi(\varrho)+\psi(\varrho) m^{i} \varphi\left(\frac{\varsigma}{m^{i}}\right)\right] \\
& \leq \frac{1}{n^{2}} \sum_{i=1}^{n}(1-s \kappa)^{2 i} \psi(\varrho) \varphi(\varrho)+\frac{1}{n^{2}} \sum_{i=1}^{n}\left[1-(s(1-\kappa))^{i}\right]^{2} m^{2 i} \psi\left(\frac{\varsigma}{m^{i}}\right) \varphi\left(\frac{\varsigma}{m^{i}}\right) \\
& +\frac{1}{n^{2}} \sum_{i=1}^{n}\left(1-(s(1-\kappa))^{i}\right)(1-(s \kappa))^{i}\left[m^{2 i} \psi\left(\frac{\varsigma}{m^{i}}\right) \varphi\left(\frac{\varsigma}{m^{i}}\right)+\psi(\varrho) \varphi(\varrho)\right]
\end{aligned}
$$




$$
\begin{aligned}
& =\left[\frac{1}{n} \sum_{i=1}^{n}\left(1-(s \kappa)^{i}\right) \psi(\varrho) \varphi(\varrho)+\frac{1}{n} \sum_{i=1}^{n}\left(1-(s(1-\kappa))^{i}\right) m^{2 i} \psi\left(\frac{\varsigma}{m^{i}}\right) \varphi\left(\frac{\varsigma}{m^{i}}\right)\right] \\
& \times\left(\frac{1}{n} \sum_{i=1}^{n}\left(1-(s(1-\kappa))^{i}\right)+\frac{1}{n} \sum_{i=1}^{n}\left(1-(s \kappa)^{i}\right)\right) \\
& \leq \frac{1}{n} \sum_{i=1}^{n}\left(1-(s \kappa)^{i}\right) \psi(\varrho) \varphi(\varrho)+\frac{1}{n} \sum_{i=1}^{n}\left(1-(s(1-\kappa))^{i}\right) m^{2 i} \psi\left(\frac{\varsigma}{m^{i}}\right) \varphi\left(\frac{\varsigma}{m^{i}}\right) .
\end{aligned}
$$

This completes the proof.

Remark 4. Taking $n=m=1$ and $\eta(\varsigma, \varrho)=\varsigma-\varrho$ in Theorem 7 , then

$$
\psi(\kappa \varsigma+(1-\kappa) \varrho) \varphi(\kappa \varsigma+(1-\kappa) \varrho) \leq[1-(s(1-\kappa))] \psi(\varsigma) \varphi(\varsigma)+[1-\varsigma \kappa] \psi(\varrho) \varphi(\varrho) .
$$

\section{Hermite-Hadamard-Type Inequality via Generalized Preinvex Function}

The principal intention of this section is to establish a novel version of the HermiteHadamard-type inequality in the setting of the newly discussed concept.

Theorem 10. Let $\mathbb{A}^{\circ} \subseteq \mathbb{R}$ be an open invex subset with respect to $\eta: \mathbb{A}^{\circ} \times \mathbb{A}^{\circ} \times(0,1] \rightarrow \mathbb{R}$, and let $\varsigma, \varrho \in \mathbb{A}^{\circ}, \varsigma<\varrho$ with $\varrho+\eta(\varsigma, \varrho) \leq \varrho$. Suppose that $\psi:[\varrho+\eta(\varsigma, \varrho), \varrho] \rightarrow[0, \infty], m \in(0,1]$, and satisfies Condition-C; then, the following Hermite-Hadamard-type inequalities hold:

$$
\begin{gathered}
\psi\left(\varrho+\frac{1}{2} \eta(\varsigma, \varrho)\right) \leq \frac{\frac{1}{n} \sum_{i=1}^{n}\left(1-\frac{s}{2}\right)^{i}}{\eta(\varsigma, \varrho)}\left[\int_{\varrho}^{\varrho+\eta(\varsigma, \varrho)} m^{i} \psi\left(\frac{x}{m^{i}}\right) d x+\int_{\frac{m \varrho+\eta(s, m \varrho)}{m}}^{\varrho} \psi(x) d x\right] \\
\leq \frac{1}{n} \sum_{i=1}^{n}(2-s)^{i}\left[\psi(\varrho)+m^{i} \psi\left(\frac{\varsigma}{m^{i}}\right)\right] .
\end{gathered}
$$

Proof. Since $\varsigma, \varrho \in \mathbb{A}^{\circ}$, and $\mathbb{A}^{\circ}$ is an invex set with respect to $\eta$, for every $m \in(0,1]$ and $\kappa \in[0,1]$, we have $\varrho+\kappa \eta(\varsigma, \varrho) \in \mathbb{A}^{\circ}$.

Using Definition 7 for $\kappa=\frac{1}{2}$, one has

$$
\begin{aligned}
& \psi(y+\kappa \eta(x, y)) \leq \frac{1}{n} \sum_{i=1}^{n}(1-(s \kappa))^{i} \psi(y)+\frac{1}{n} \sum_{i=1}^{n}\left(1-(s(1-\kappa))^{i}\right) m^{i} \psi\left(\frac{x}{m^{i}}\right) \\
& \psi\left(y+\frac{1}{2} \eta(x, y)\right) \leq \frac{1}{n} \sum_{i=1}^{n}\left(1-\frac{s}{2}\right)^{i}\left[m^{i} \psi\left(\frac{x}{m^{i}}\right)+\psi(y)\right]
\end{aligned}
$$

Choosing $x=\varrho+\kappa \eta(\varsigma, \varrho)$ and $m y=m \varrho+(1-\kappa) \eta(\varsigma, m \varrho)$ in the last inequality, one obtains

$$
\psi\left(y+\frac{1}{2} \eta(x, y)\right)=\psi\left(\varrho+(1-\kappa) \eta(\varsigma, \varrho)+\frac{1}{2} \eta(\varrho+\kappa \eta(\varsigma, \varrho), \varrho+(1-\kappa) \eta(\varsigma, \varrho))\right)
$$

Now, by applying the extended Condition-C, one has

$$
\begin{gathered}
\eta(\varrho+\kappa \eta(\varsigma, \varrho), \varrho+(1-\kappa) \eta(\varsigma, \varrho))=(\kappa-1+\kappa) \eta(\varsigma, \varrho) \\
\eta(\varrho+\kappa \eta(\varsigma, \varrho), \varrho+(1-\kappa) \eta(\varsigma, \varrho))=(2 \kappa-1) \eta(\varsigma, \varrho)
\end{gathered}
$$

Consequently, Equation (9) becomes

$$
\begin{gathered}
\psi\left(y+\frac{1}{2} \eta(x, y)\right)=\psi\left(\varrho+(1-\kappa) \eta(\varsigma, \varrho)+\frac{1}{2}(2 \kappa-1) \eta(\varsigma, \varrho)\right) \\
\psi\left(y+\frac{1}{2} \eta(x, y)\right)=\psi\left(\varrho+\left(1-\kappa+\kappa-\frac{1}{2}\right) \eta(\varsigma, \varrho)\right)
\end{gathered}
$$




$$
\psi\left(y+\frac{1}{2} \eta(x, y)\right)=\psi\left(\varrho+\frac{1}{2} \eta(\varsigma, \varrho)\right)
$$

Now,

$$
\begin{aligned}
& \psi\left(\varrho+\frac{1}{2} \eta(\varsigma, \varrho)\right) \\
& \leq \frac{1}{n} \sum_{i=1}^{n}\left(1-\frac{s}{2}\right)^{i}\left[\int_{0}^{1} m^{i} \psi\left(\frac{\varrho+\kappa \eta(\varsigma, \varrho)}{m^{i}}\right) d \kappa+\int_{0}^{1} \psi\left(\frac{m \varrho+(1-\kappa) \eta(\varsigma, m \varrho)}{m}\right) d \kappa\right] \\
& \leq \frac{1}{n} \sum_{i=1}^{n}\left(1-\frac{s}{2}\right)^{i} \frac{1}{\eta(\varsigma, \varrho)}\left[\int_{\varrho}^{\varrho+\eta(\varsigma, \varrho)} m^{i} \psi\left(\frac{x}{m^{i}}\right) d x+\int_{\frac{m \varrho+\eta(\zeta, m \varrho)}{m}}^{\varrho} \psi(x) d x\right] .
\end{aligned}
$$

This completes the proof of the first part of the inequality. To prove the second part, we need the definition of generalized s-type $m$-preinvexity:

$$
\begin{aligned}
& \frac{1}{\eta(\varsigma, \varrho)}\left[\int_{\varrho}^{\varrho+\eta(\varsigma, \varrho)} m^{i} \psi\left(\frac{x}{m^{i}}\right) d x+\int_{\frac{m \varrho+\eta(\varsigma, \varrho)}{m}}^{\varrho} \psi(x) d x\right] \\
& \leq\left[\int_{0}^{1} \psi(\varrho+\kappa \eta(\varsigma, \varrho)) d \kappa+\int_{0}^{1} \psi\left(\frac{m \varrho+(1-\kappa) \eta(\varsigma, m \varrho)}{m}\right) d \kappa\right] \\
& \leq \int_{0}^{1} \frac{1}{n} \sum_{i=1}^{n}\left(1-(s \kappa)^{i}\right) \psi(\varrho) d \kappa+\int_{0}^{1} \frac{1}{n} \sum_{i=1}^{n}\left(1-(s(1-\kappa))^{i}\right) m^{i} \psi\left(\frac{\varsigma}{m^{i}}\right) d \kappa \\
& +\int_{0}^{1} \frac{1}{n} \sum_{i=1}^{n}\left(1-(s(1-\kappa))^{i}\right) \psi(\varrho) d \kappa+\int_{0}^{1} \frac{1}{n} \sum_{i=1}^{n}\left(1-(s \kappa)^{i}\right) m^{i} \psi\left(\frac{\varsigma}{m^{i}}\right) d \kappa \\
& \leq \frac{1}{n} \sum_{i=1}^{n}\left(\frac{2-s}{2}\right)^{i}\left[\psi(\varrho)+\psi(\varrho)+m^{i} \psi\left(\frac{\varsigma}{m^{i}}\right)+m^{i} \psi\left(\frac{\varsigma}{m^{i}}\right)\right] \\
& \leq \frac{1}{n} \sum_{i=1}^{n}(2-s)^{i}\left[\psi(\varrho)+m^{i} \psi\left(\frac{\varsigma}{m^{i}}\right)\right] .
\end{aligned}
$$

This completes the proof of the desired inequality.

Corollary 1. If we put $n=s=m=1$ and $\eta(s, \varrho)=\varsigma-\varrho$ in Theorem 10, it reduces to the Hermite-Hadamard inequality given in [5].

Remark 5. If we put $\eta(\varsigma, \varrho)=\varsigma-\varrho$ in Theorem 10, then we obtain the following inequality:

$$
\begin{array}{r}
\psi\left(\frac{\varsigma+\varrho}{2}\right) \leq \frac{\frac{1}{n} \sum_{i=1}^{n}\left(1-\frac{\varsigma}{2}\right)^{i}}{(\varsigma-\varrho)}\left[\int_{\varrho}^{\varsigma} m^{i} \psi\left(\frac{x}{m^{i}}\right) d x+\int_{\frac{\varsigma}{m}}^{\varrho} \psi(x) d x\right] \\
\leq \frac{1}{n} \sum_{i=1}^{n}(2-s)^{i}\left[\psi(\varrho)+m^{i} \psi\left(\frac{\varsigma}{m^{i}}\right)\right] .
\end{array}
$$

Remark 6. If we put $m=1$ and $\eta(\varsigma, \varrho)=\varsigma-\varrho$ in Theorem 10 , then we obtain the following inequality:

$$
\frac{1}{2} \psi\left(\frac{\varsigma+\varrho}{2}\right) \leq \frac{\frac{1}{n} \sum_{i=1}^{n}\left(1-\frac{s}{2}\right)^{i}}{(\varrho-\varsigma)} \int_{\zeta}^{\varrho} \psi(x) d x \leq \frac{1}{2 n} \sum_{i=1}^{n}(2-s)^{i}[\psi(\varrho)+\psi(\varsigma)] .
$$

\section{Refinements of Hermite-Hadamard-Type Inequality}

To present our main results as the refinements of the Hermite-Hadamard-type inequality using generalized s-type preinvex functions, we need the following well known lemma:

Lemma 3 ([31]). Let $\psi: \mathbb{A}^{\circ} \subseteq \mathbb{R} \rightarrow \mathbb{R}$ be a differentiable mapping on $\mathbb{A}^{\circ}, \varsigma, \varrho \in \mathbb{A}^{\circ}$ with $\varsigma<\varrho$. If $\psi^{\prime} \in L[\varsigma, \varrho]$, then 


$$
-\frac{\psi(\varrho)+\psi(\varrho+\eta(\varsigma, \varrho))}{2}+\frac{1}{\eta(\varsigma, \varrho)} \int_{\varrho}^{\varrho+\eta(\varsigma, \varrho)} \psi(x) d x=\frac{\eta(\varsigma, \varrho)}{2} \int_{0}^{1}(1-2 \kappa) \psi^{\prime}(\varrho+\kappa \eta(\varsigma, \varrho)) d \kappa .
$$

Theorem 11. Let $\mathbb{A}^{\circ} \subseteq \mathbb{R}$ be an open invex subset with respect to $\eta: \mathbb{A}^{\circ} \times \mathbb{A}^{\circ} \rightarrow \mathbb{R}$ and $\varsigma, \varrho \in \mathbb{A}^{\circ}$ with $\varrho+\eta(\varsigma, \varrho) \leq \varrho$. Suppose that $\psi: \mathbb{A}^{\circ} \rightarrow \mathbb{R}$ is a differentiable function such that $\psi^{\prime} \in L[\varrho+\eta(\varsigma, \varrho), \varrho]$. If $\left|\psi^{\prime}\right|$ is a generalized s-type m-preinvex function on $[\varrho+\eta(\varsigma, \varrho), \varrho]$, then for $\kappa \in[0,1]$ and $s \in[0,1]$, the following inequality

$$
\begin{aligned}
& \left|\frac{\psi(\varrho)+\psi(\varrho+\eta(\varsigma, \varrho))}{2}-\frac{1}{\eta(\varsigma, \varrho)} \int_{\varrho}^{\varrho+\eta(\varsigma, \varrho)} \psi(x) d x\right| \\
& \leq \frac{|\eta(\varsigma, \varrho)|}{n} \sum_{i=1}^{n}\left[\frac{\left(i^{2}+3 i+2\right) 2^{i}-\left(1+3^{i} i\right) 2 s^{i}}{2^{i+1}(i+1)(i+2)}\right] A\left(m^{i}\left|\psi^{\prime}\left(\frac{\varsigma}{m^{i}}\right)\right|,\left|\psi^{\prime}(\varrho)\right|\right),
\end{aligned}
$$

holds, where $A(.,$.$) is the arithmetic mean.$

Proof. Let $\zeta, \varrho \in \mathbb{A}^{\circ}$. Since $\mathbb{A}^{\circ}$ is an invex set with respect to $\eta$, for any $\kappa \in[0,1]$, we have $\varrho+\kappa \eta(\varsigma, \varrho) \in \mathbb{A}^{\circ}$.

Using Lemma 3, the generalized s-type $m$-preinvexity of $\left|\psi^{\prime}\right|$, and the properties of modulus, we have

$$
\begin{aligned}
& \left|\frac{\psi(\varrho)+\psi(\varrho+\eta(\varsigma, \varrho))}{2}-\frac{1}{\eta(\varsigma, \varrho)} \int_{\varrho}^{\varrho+\eta(\varsigma, \varrho)} \psi(x) d x\right| \\
& \leq\left|\frac{\eta(\varsigma, \varrho)}{2} \int_{0}^{1}(1-2 \kappa) \psi^{\prime}(\varrho+\kappa \eta(\varsigma, \varrho)) d \kappa\right| \\
& \left.\leq \frac{|\eta(\varsigma, \varrho)|}{2} \int_{0}^{1}|1-2 \kappa|\left(\frac{1}{n} \sum_{i=1}^{n}[1-(s \kappa))^{i}\right]\left|\psi^{\prime}(\varrho)\right|+\frac{1}{n} \sum_{i=1}^{n}\left[1-(s(1-\kappa))^{i}\right] m^{i}\left|\psi^{\prime}\left(\frac{\varsigma}{m^{i}}\right)\right|\right) d \kappa \\
& \leq \frac{|\eta(\varsigma, \varrho)|}{2 n}\left(\left|\psi^{\prime}(\varrho)\right| \sum_{i=1}^{n} \int_{0}^{1}|1-2 \kappa|[1-(s \kappa))^{i}\right] d \kappa \\
& \left.\quad+\sum_{i=1}^{n} m^{i}\left|\psi^{\prime}\left(\frac{\varsigma}{m^{i}}\right)\right| \int_{0}^{1}|1-2 \kappa|\left[1-(s(1-\kappa))^{i}\right] d \kappa\right) \\
& \leq \frac{|\eta(\varsigma, \varrho)|}{2 n}\left(\left|\psi^{\prime}(\varrho)\right| \sum_{i=1}^{n}\left[\frac{\left(i^{2}+3 i+2\right) 2^{i}-\left(1+2^{i} i\right) 2 s^{i}}{2^{i+1}(i+1)(i+2)}\right]\right. \\
& \left.\quad+\sum_{i=1}^{n}\left|m^{i} \psi^{\prime}\left(\frac{\varsigma}{m^{i}}\right)\right|\left[\frac{\left(i^{2}+3 i+2\right) 2^{i}-\left(1+2^{i} i\right) 2 s^{i}}{2^{i+1}(i+1)(i+2)}\right]\right) \\
& \leq \frac{|\eta(\varsigma, \varrho)|}{n} \sum_{i=1}^{n}\left[\frac{\left(i^{2}+3 i+2\right) 2^{i}-\left(1+2^{i} i\right) 2 s^{i}}{2^{i+1}(i+1)(i+2)}\right] A\left(m^{i}\left|\psi^{\prime}\left(\frac{\varsigma}{m^{i}}\right)\right|,\left|\psi^{\prime}(\varrho)\right|\right) .
\end{aligned}
$$

This completes the proof of the desired result.

Corollary 2. If we put $m=n=1$ and $s=1$ in Theorem 11, then we obtain Theorem (2.1) in [31].

Corollary 3. If we put $m=1$ and $\eta(\varsigma, \varrho)=\varsigma-\varrho$ in Theorem 11, we obtain inequality (4.1) in [27].

Corollary 4. If we put $m=n=s=1$ and $\eta(\varsigma, \varrho)=\varsigma-\varrho$ in Theorem 11, then we obtain Corollary 1 in [27].

Theorem 12. Let $\mathbb{A}^{\circ} \subseteq \mathbb{R}$ be an open invex subset with respect to $\eta: \mathbb{A}^{\circ} \times \mathbb{A}^{\circ} \rightarrow \mathbb{R}$ and $\varsigma, \varrho \in \mathbb{A}^{\circ}$ with $\varrho+\eta(\varsigma, \varrho) \leq \varrho, q>1, \frac{1}{p}+\frac{1}{q}=1$. Suppose that $\psi: \mathbb{A}^{\circ} \rightarrow \mathbb{R}$ is a differentiable 
function such that $\psi^{\prime} \in L[\varrho+\eta(\varsigma, \varrho), \varrho]$. If $\left|\psi^{\prime}\right|$ is a generalized s-type m-preinvex function on $[\varrho+\eta(\varsigma, \varrho), \varrho]$, then for $\kappa \in[0,1]$ and $s \in[0,1]$, the following inequality

$$
\begin{aligned}
& \left|\frac{\psi(\varrho)+\psi(\varrho+\eta(\varsigma, \varrho))}{2}-\frac{1}{\eta(\varsigma, \varrho)} \int_{\varrho}^{\varrho+\eta(\varsigma, \varrho)} \psi(x) d x\right| \\
& \leq \frac{|\eta(\varsigma, \varrho)|}{2 \sqrt[q]{n}}\left(\frac{1}{p+1}\right)^{\frac{1}{p}}\left(2 \sum_{i=1}^{n} \frac{i+1-s^{i}}{i+1}\right)^{\frac{1}{q}} A^{\frac{1}{q}}\left(m^{i}\left|\psi^{\prime}\left(\frac{\varsigma}{m^{i}}\right)\right|^{q},\left|\psi^{\prime}(\varrho)\right|^{q}\right),
\end{aligned}
$$

holds, where $A(.,$.$) is the arithmetic mean.$

Proof. Let $\zeta, \varrho \in \mathbb{A}^{\circ}$. Since $\mathbb{A}^{\circ}$ is an invex set with respect to $\eta$, for any $\kappa \in[0,1]$, we have $\varrho+\kappa \eta(\varsigma, \varrho) \in \mathbb{A}^{\circ}$.

From Lemma 3, Hölder's integral inequality, the generalized s-type $m$-preinvexity of $\left|\psi^{\prime}\right| q$, and the properties of modulus, we have

$$
\begin{aligned}
& \left|\frac{\psi(\varrho)+\psi(\varrho+\eta(\varsigma, \varrho))}{2}-\frac{1}{\eta(\varsigma, \varrho)} \int_{\varrho}^{\varrho+\eta(\varsigma, \varrho)} \psi(x) d x\right| \\
& \leq\left|\frac{\eta(\varsigma, \varrho)}{2} \int_{0}^{1}(1-2 \kappa) \psi^{\prime}(\varrho+\kappa \eta(\varsigma, \varrho)) d \kappa\right| \\
& \leq \frac{|\eta(\varsigma, \varrho)|}{2}\left(\int_{0}^{1}|1-2 \kappa|^{p}\right)^{\frac{1}{p}}\left(\int_{0}^{1}\left|\psi^{\prime}(\varrho+\kappa \eta(\varsigma, \varrho))\right|^{q} d \kappa\right)^{\frac{1}{q}} \\
& \leq \frac{|\eta(\varsigma, \varrho)|}{2 \sqrt[q]{n}}\left(\frac{1}{p+1}\right)^{\frac{1}{p}}\left(\left|\psi^{\prime}(\varrho)\right|^{q} \int_{0}^{1} \sum_{i=1}^{n}\left(1-(s \kappa)^{i}\right) d \kappa\right. \\
& \left.\quad+\int_{0}^{1} \sum_{i=1}^{n} m^{i}\left|\psi^{\prime}\left(\frac{\varsigma}{m^{i}}\right)\right|^{q}\left[1-(s(1-\kappa))^{i}\right] d \kappa\right)^{\frac{1}{q}} \\
& \leq \frac{|\eta(\varsigma, \varrho)|}{2 \sqrt[q]{n}}\left(\frac{1}{p+1}\right)^{\frac{1}{p}}\left(2 \sum_{i=1}^{n} \frac{i+1-s^{i}}{i+1}\right)^{\frac{1}{q}} A^{\frac{1}{q}}\left(m^{i}\left|\psi^{\prime}\left(\frac{\varsigma}{m^{i}}\right)\right|^{q},\left|\psi^{\prime}(\varrho)\right|^{q}\right) .
\end{aligned}
$$

This completes the proof of the desired result.

Corollary 5. If we put $m=n=s=1$ in Theorem 12, then we obtain Theorem (2.2) in [31].

Corollary 6. If we put $m=s=1$ and $\eta(\varsigma, \varrho)=\varsigma-\varrho$ in Theorem 12 , then we obtain inequality (4.2) in [27].

Corollary 7. If we put $m=n=s=1$ and $\eta(\varsigma, \varrho)=\varsigma-\varrho$ in Theorem 12, then we obtain Corollary 2 in [27].

Theorem 13. Suppose $\mathbb{A}^{\circ} \subseteq \mathbb{R}$ is an open invex subset with respect to $\eta: \mathbb{A}^{\circ} \times \mathbb{A}^{\circ} \rightarrow \mathbb{R}$ and $\varsigma, \varrho \in \mathbb{A}^{\circ}$ with $\varrho+\eta(\varsigma, \varrho) \leq \varrho, q \geq 1$. Suppose that $\psi: \mathbb{A}^{\circ} \rightarrow \mathbb{R}$ is a differentiable function such that $\psi^{\prime} \in L[\varrho+\eta(\varsigma, \varrho), \varrho]$. If $\left|\psi^{\prime}\right|$ is a generalized s-type m-preinvex function on $[\varrho+\eta(\varsigma, \varrho), \varrho]$, then for $\kappa \in[0,1]$ and $s \in[0,1]$, the following inequality

$$
\begin{aligned}
& \left|\frac{\psi(\varrho)+\psi(\varrho+\eta(\varsigma, \varrho))}{2}-\frac{1}{\eta(\varsigma, \varrho)} \int_{\varrho}^{\varrho+\eta(\varsigma, \varrho)} \psi(x) d x\right| \\
& \leq \frac{|\eta(\varsigma, \varrho)|}{2 \sqrt[q]{n}}\left(\frac{1}{2}\right)^{1-\frac{2}{q}}\left(\sum_{i=1}^{n}\left[\frac{\left(i^{2}+3 i+2\right) 2^{i}-\left(1+2^{i} i\right) 2 s^{i}}{2^{i+1}(i+1)(i+2)}\right]\right)^{\frac{1}{q}} A^{\frac{1}{q}}\left(m^{i}\left|\psi^{\prime}\left(\frac{\varsigma}{m^{i}}\right)\right|^{q},\left|\psi^{\prime}(\varrho)\right|^{q}\right),
\end{aligned}
$$

holds, where $A(.,$.$) is the arithmetic mean.$ 
Proof. Let $\zeta, \varrho \in \mathbb{A}^{\circ}$. Since $\mathbb{A}^{\circ}$ is an invex set with respect to $\eta$, for any $\kappa \in[0,1]$, we have $\varrho+\kappa \eta(\varsigma, \varrho) \in \mathbb{A}^{\circ}$.

Suppose that $q>1$. Using Lemma 3, the power mean inequality, the generalized s-type $m$-preinvexity of $\left|\psi^{\prime}\right|^{q}$, and the properties of modulus, we have

$$
\begin{aligned}
& \left|\frac{\psi(\varrho)+\psi(\varrho+\eta(\varsigma, \varrho))}{2}-\frac{1}{\eta(\zeta, \varrho)} \int_{\varrho}^{\varrho+\eta(\varsigma, \varrho)} \psi(x) d x\right| \\
& \leq\left|\frac{\eta(\varsigma, \varrho)}{2} \int_{0}^{1}(1-2 \kappa) \psi^{\prime}(\varrho+\kappa \eta(\varsigma, \varrho)) d \kappa\right| \\
& \leq \frac{|\eta(\varsigma, \varrho)|}{2}\left(\int_{0}^{1}|1-2 \kappa| d \kappa\right)^{1-\frac{1}{q}}\left(\int_{0}^{1}|1-2 \kappa|\left|\psi^{\prime}(\varrho+\kappa \eta(\varsigma, \varrho))\right|^{q} d \kappa\right)^{\frac{1}{q}} \\
& \leq \frac{|\eta(\zeta, \varrho)|}{2 \sqrt[q]{n}}\left(\frac{1}{2}\right)^{1-\frac{1}{q}} \\
& \left.\times\left(\int_{0}^{1}|1-2 \kappa|\left[\sum_{i=1}^{n}[1-(s \kappa))^{i}\right]\left|\psi^{\prime}(\varrho)\right|^{q}+\sum_{i=1}^{n}\left[1-(s(1-\kappa))^{i}\right]\left|\psi^{\prime}(\varsigma)\right|^{q}\right] d \kappa\right)^{\frac{1}{q}} \\
& \leq \frac{|\eta(\zeta, \varrho)|}{2 \sqrt[q]{n}}\left(\frac{1}{2}\right)^{1-\frac{1}{q}} \\
& \left.\times\left(\left|\psi^{\prime}(\varrho)\right|^{q} \int_{0}^{1}|1-2 \kappa| \sum_{i=1}^{n}[1-(s \kappa))^{i}\right] d \kappa+\int_{0}^{1}|1-2 \kappa| \sum_{i=1}^{n} m^{i}\left|\psi^{\prime}\left(\frac{\zeta}{m^{i}}\right)\right|^{q}\left[1-(s(1-\kappa))^{i}\right] d \kappa\right)^{\frac{1}{q}} \\
& =\frac{|\eta(\zeta, \varrho)|}{2 \sqrt[q]{n}}\left(\frac{1}{2}\right)^{1-\frac{2}{q}}\left(\sum_{i=1}^{n}\left[\frac{\left(i^{2}+3 i+2\right) 2^{i}-\left(1+2^{i} i\right) 2 s^{i}}{2^{i+1}(i+1)(i+2)}\right]\right)^{\frac{1}{q}} A^{\frac{1}{q}}\left(m^{i}\left|\psi^{\prime}\left(\frac{\varsigma}{m^{i}}\right)\right|^{q},\left|\psi^{\prime}(\varrho)\right|^{q}\right) .
\end{aligned}
$$

Furthermore, for $q=1$, Using the same procedure step by step as in Theorem 11, we are led to the required result.

Corollary 8. If we put $n=m=1$ and $s=1$ in Theorem 13, then

$$
\left|\frac{\psi(\varrho)+\psi(\varrho+\eta(\varsigma, \varrho))}{2}-\frac{1}{\eta(\varsigma, \varrho)} \int_{\varrho}^{\varrho+\eta(\varsigma, \varrho)} \psi(x) d x\right| \leq \frac{\eta(\varsigma, \varrho)}{4} A^{\frac{1}{q}}\left[\left|\psi^{\prime}(\varsigma)\right|^{q},\left|\psi^{\prime}(\varrho)\right|^{q}\right] .
$$

Corollary 9. If we put $s=1$ and $\eta(\varsigma, \varrho)=\varsigma-\varrho$ in Theorem 13, we obtain inequality (4.3) in [27].

Corollary 10. If we put $n=1, s=1$, and $\eta(\varsigma, \varrho)=\varsigma-\varrho$ in Theorem 13, then we obtain Corollary 4 in [27].

Theorem 14. Let $\mathbb{A}^{\circ} \subseteq \mathbb{R}$ be an open invex subset with respect to $\eta: \mathbb{A}^{\circ} \times \mathbb{A}^{\circ} \rightarrow \mathbb{R}$ and $\varsigma, \varrho \in \mathbb{A}^{\circ}$ with $\varrho+\eta(\varsigma, \varrho) \leq \varrho, q>1, \frac{1}{p}+\frac{1}{q}=1$. Suppose that $\psi: \mathbb{A}^{\circ} \rightarrow \mathbb{R}$ is a differentiable function such that $\psi^{\prime} \in L[\varrho+\eta(\varsigma, \varrho), \varrho]$. If $\left|\psi^{\prime}\right|$ is a generalized s-type m-preinvex function on $[\varrho+\eta(\varsigma, \varrho), \varrho]$, then for $\kappa \in[0,1]$ and $s \in[0,1]$, the following inequality

$$
\begin{aligned}
& \left|\frac{\psi(\varrho)+\psi(\varrho+\eta(\varsigma, \varrho))}{2}-\frac{1}{\eta(\varsigma, \varrho)} \int_{\varrho}^{\varrho+\eta(s, \varrho)} \psi(x) d x\right| \\
& \leq \frac{|\eta(\varsigma, \varrho)|}{2 \sqrt[q]{n}}\left(\frac{1}{2(p+1)}\right)^{\frac{1}{p}} \\
& \times\left\{\left(m^{i}\left|\psi^{\prime}\left(\frac{\varsigma}{m^{i}}\right)\right|^{q} \sum_{i=1}^{n} \frac{i+2-2 s^{i}}{2(i+2)}+\left|\psi^{\prime}(\varrho)\right|^{q} \sum_{i=1}^{n} \frac{i^{2}+3 i+2-2 s^{i}}{2(i+1)(i+2)}\right)^{\frac{1}{q}}\right. \\
& \left.+\left(m^{i}\left|\psi^{\prime}\left(\frac{\varsigma}{m^{i}}\right)\right|^{q} \sum_{i=1}^{n} \frac{i^{2}+3 i+2-2 s^{i}}{2(i+1)(i+2)}+\left|\psi^{\prime}(\varrho)\right|^{q} \sum_{i=1}^{n} \frac{i+2-2 s^{i}}{2(i+2)}\right)^{\frac{1}{q}}\right\},
\end{aligned}
$$


holds.

Proof. Suppose that $\varsigma, \varrho \in \mathbb{A}^{\circ}$. Since $\mathbb{A}^{\circ}$ is an invex set with respect to $\eta$, for any $\kappa \in[0,1]$, we have $\varrho+\kappa \eta(\varsigma, \varrho) \in \mathbb{A}^{\circ}$.

From Lemma 3, Hölder-İscan integral inequality, the generalized $s$-type $m$-preinvexity of $\left|\psi^{\prime}\right|^{q}$, and the properties of modulus, we have

$$
\begin{aligned}
& \left|\frac{\psi(\varrho)+\psi(\varrho+\eta(\varsigma, \varrho))}{2}-\frac{1}{\eta(\varsigma, \varrho)} \int_{\varrho}^{\varrho+\eta(\varsigma, \varrho)} \psi(x) d x\right| \\
& \leq \frac{|\eta(\varsigma, \varrho)|}{2} \int_{0}^{1}|1-2 \kappa|\left|\psi^{\prime}(\varrho+\kappa \eta(\varsigma, \varrho))\right| d \kappa \\
& \leq \frac{|\eta(\varsigma, \varrho)|}{2}\left(\int_{0}^{1}(1-\kappa)|1-2 \kappa|^{p} d \kappa\right)^{\frac{1}{p}}\left(\int_{0}^{1}(1-\kappa)\left|\psi^{\prime}(\varrho+\kappa \eta(\varsigma, \varrho))\right|^{q} d \kappa\right)^{\frac{1}{q}} \\
& +\frac{|\eta(\varsigma, \varrho)|}{2}\left(\int_{0}^{1} \kappa|1-2 \kappa|^{p} d \kappa\right)^{\frac{1}{p}}\left(\int_{0}^{1} \kappa\left|\psi^{\prime}(\varrho+\kappa \eta(\varsigma, \varrho))\right|^{q} d \kappa\right)^{\frac{1}{q}} \\
& \leq \frac{|\eta(\varsigma, \varrho)|}{2 \sqrt[q]{n}}\left(\frac{1}{2(p+1)}\right)^{\frac{1}{p}} \\
& \times\left(\left|\psi^{\prime}(\varrho)\right|^{q} \sum_{i=1}^{n} \int_{0}^{1}(1-\kappa)\left(1-(s \kappa)^{i}\right) d \kappa+\sum_{i=1}^{n} \int_{0}^{1}(1-\kappa) m^{i}\left|\psi^{\prime}\left(\frac{\varsigma}{m^{i}}\right)\right|^{q}\left[1-(s(1-\kappa))^{i}\right] d \kappa\right)^{\frac{1}{q}} \\
& +\frac{|\eta(\varsigma, \varrho)|}{2 \sqrt[q]{n}}\left(\frac{1}{2(p+1)}\right)^{\frac{1}{p}} \\
& \times\left(\left|\psi^{\prime}(\varrho)\right|^{q} \sum_{i=1}^{n} \int_{0}^{1} \kappa\left(1-(s \kappa)^{i}\right) d \kappa+\sum_{i=1}^{n} \int_{0}^{1} m^{i}\left|\psi^{\prime}\left(\frac{\varsigma}{m^{i}}\right)\right|^{q} \kappa\left[1-(s(1-\kappa))^{i}\right] d \kappa\right)^{\frac{1}{q}} \\
& \leq \frac{|\eta(\varsigma, \varrho)|}{2 \sqrt[\frac{1}{n}]{n}}\left(\frac{1}{2(p+1)}\right)^{\frac{1}{p}}\left\{\left(m^{i}\left|\psi^{\prime}\left(\frac{\varsigma}{m^{i}}\right)\right|^{q} \sum_{i=1}^{n} \frac{i+2-2 s^{i}}{2(i+2)}+\left|\psi^{\prime}(\varrho)\right|^{q} \sum_{i=1}^{n} \frac{i^{2}+3 i+2-2(i+1)(i+2)}{2 s^{i}}\right)^{\frac{1}{q}}\right. \\
& +\left(m^{i} \mid \psi^{\prime}\left(\left.\frac{\varsigma}{m^{i}}\right|^{q} \sum_{i=1}^{n} \frac{i^{2}+3 i+2-2 s^{i}}{2(i+1)(i+2)}+\left|\psi^{\prime}(\varrho)\right|^{q} \sum_{i=1}^{n} \frac{i+2-2 s^{i}}{2(i+2)}\right)^{\frac{1}{q}}\right\} .
\end{aligned}
$$

This completes the proof of the desired result.

Corollary 11. If we put $n=m=1$ and $s=1$ in Theorem 14 , then

$$
\begin{aligned}
& \left|\frac{\psi(\varrho)+\psi(\varrho+\eta(\varsigma, \varrho))}{2}-\frac{1}{\eta(\varsigma, \varrho)} \int_{\varrho}^{\varrho+\eta(\varsigma, \varrho)} \psi(x) d x\right| \\
& \leq \frac{|\eta(\varsigma, \varrho)|}{4}\left(\frac{1}{p+1}\right)^{\frac{1}{p}}\left[\left(\frac{\left|\psi^{\prime}(\varsigma)\right|^{q}}{3}+\frac{2\left|\psi^{\prime}(\varrho)\right|^{q}}{3}\right)^{\frac{1}{q}}+\left(\frac{2\left|\psi^{\prime}(\varsigma)\right|^{q}}{3}+\frac{\left|\psi^{\prime}(\varrho)\right|^{q}}{3}\right)^{\frac{1}{q}}\right] .
\end{aligned}
$$

Corollary 12. If we put $s=m=1$ and $\eta(\varsigma, \varrho)=\varsigma-\varrho$ in Theorem 14 , we obtain inequality (4.4) in [27].

Corollary 13. If we put $n=m=1, s=1$, and $\eta(\varsigma, \varrho)=\varsigma-\varrho$ in Theorem 14, then we obtain Corollary 5 in [27].

Theorem 15. Let $\mathbb{A}^{\circ} \subseteq \mathbb{R}$ be an open invex subset with respect to $\eta: \mathbb{A}^{\circ} \times \mathbb{A}^{\circ} \rightarrow \mathbb{R}$ and $\varsigma, \varrho \in \mathbb{A}^{\circ}$ with $\varrho+\eta(\varsigma, \varrho) \leq \varrho, q \geq 1$. Suppose that $\psi: \mathbb{A}^{\circ} \rightarrow \mathbb{R}$ is a differentiable function such 
that $\psi^{\prime} \in \mathcal{L}[\varrho+\eta(\varsigma, \varrho), \varrho]$. If $\left|\psi^{\prime}\right|$ is a generalized s-type m-preinvex function on $[\varrho+\eta(\varsigma, \varrho), \varrho]$, then for $\kappa \in[0,1]$ and $s \in[0,1]$, the following inequality

$$
\begin{aligned}
& \left|\frac{\psi(\varrho)+\psi(\varrho+\eta(\varsigma, \varrho))}{2}-\frac{1}{\eta(\varsigma, \varrho)} \int_{\varrho}^{\varrho+\eta(\varsigma, \varrho)} \psi(x) d x\right| \\
& \leq \frac{|\eta(\varsigma, \varrho)|}{2 \sqrt[q]{n}}\left(\frac{1}{2}\right)^{2-\frac{2}{q}}\left\{\left(\sum_{i=1}^{n} m^{i}\left|\psi^{\prime}\left(\frac{\varsigma}{m^{i}}\right)\right|^{q} k_{1}(s)+\left|\psi^{\prime}(\varrho)\right|^{q} \sum_{i=1}^{n} k_{2}(s)\right)^{\frac{1}{q}}\right. \\
& \left.+\left(\sum_{i=1}^{n} m^{i}\left|\psi^{\prime}\left(\frac{\varsigma}{m^{i}}\right)\right|^{q} k_{2}(s)+\left|\psi^{\prime}(\varrho)\right|^{q} \sum_{i=1}^{n} k_{1}(s)\right)^{\frac{1}{q}}\right\},
\end{aligned}
$$

holds, where

$$
\begin{aligned}
& \left.k_{1}(s)=\int_{0}^{1}(1-\kappa)|1-2 \kappa|\left[1-(s(1-\kappa))^{i}\right] d \kappa=\int_{0}^{1} \kappa|1-2 \kappa|[1-(s \kappa))^{i}\right] d \kappa, \\
& \left.k_{2}(s)=\int_{0}^{1} \kappa|1-2 \kappa|\left[1-(s(1-\kappa))^{i}\right] d \kappa=\int_{0}^{1}(1-\kappa)|1-2 \kappa|[1-(s \kappa))^{i}\right] d \kappa .
\end{aligned}
$$

Proof. Let $\zeta, \varrho \in \mathbb{A}^{\circ}$. Since $\mathbb{A}^{\circ}$ is an invex set with respect to $\eta$, for any $\kappa \in[0,1]$, we have $\varrho+\kappa \eta(\varsigma, \varrho) \in \mathbb{A}^{\circ}$.

Suppose that $q>1$. Using Lemma 3, the improved power-mean integral inequality, the generalized s-type $m$-preinvexity of $\left|\psi^{\prime}\right| q$, and the properties of modulus, we have

$$
\begin{aligned}
& \left|\frac{\psi(\varrho)+\psi(\varrho+\eta(\varsigma, \varrho))}{2}-\frac{1}{\eta(\varsigma, \varrho)} \int_{\varrho}^{\varrho+\eta(\zeta, \varrho)} \psi(x) d x\right| \\
& \leq \frac{|\eta(\varsigma, \varrho)|}{2} \int_{0}^{1}|1-2 \kappa|\left|\psi^{\prime}(\varrho+\kappa \eta(\varsigma, \varrho))\right| d \kappa \\
& \leq \frac{|\eta(\varsigma, \varrho)|}{2}\left(\int_{0}^{1}(1-\kappa)|1-2 \kappa| d \kappa\right)^{1-\frac{1}{q}}\left(\int_{0}^{1}(1-\kappa)|1-2 \kappa|\left|\psi^{\prime}(\varrho+\kappa \eta(\varsigma, \varrho))\right|^{q} d \kappa\right)^{\frac{1}{q}} \\
& +\frac{|\eta(\zeta, \varrho)|}{2}\left(\int_{0}^{1}(\kappa|1-2 \kappa| d \kappa)^{1-\frac{1}{q}}\left(\int_{0}^{1} \kappa|1-2 \kappa|\left|\psi^{\prime}(\varrho+\kappa \eta(\varsigma, \varrho))\right|^{q} d \kappa\right)^{\frac{1}{q}}\right. \\
& \leq \frac{|\eta(\zeta, \varrho)|}{2 \sqrt[q]{n}}\left(\frac{1}{2}\right)^{2-\frac{2}{q}}\left\{\left(\left|\psi^{\prime}(\varrho)\right|^{q} \sum_{i=1}^{n} \int_{0}^{1}(1-\kappa)|1-2 \kappa|[1-(s \kappa))^{i}\right] d \kappa\right. \\
& \left.+\sum_{i=1}^{n} \int_{0}^{1}(1-\kappa)|1-2 \kappa| m^{i}\left|\psi^{\prime}\left(\frac{\varsigma}{m^{i}}\right)\right|^{q}\left[1-(s(1-\kappa))^{i}\right] d \kappa\right)^{\frac{1}{q}} \\
& +\left(\left|\psi^{\prime}(\varrho)\right|^{q} \sum_{i=1}^{n} \int_{0}^{1} \kappa|1-2 \kappa|[1-(s \kappa))^{i}\right] d \kappa \\
& \left.\left.+\sum_{i=1}^{n} \int_{0}^{1} \kappa|1-2 \kappa| m^{i}\left|\psi^{\prime}\left(\frac{\varsigma}{m^{i}}\right)\right|^{q}\left[1-(s(1-\kappa))^{i}\right] d \kappa\right)^{\frac{1}{q}}\right\} \\
& \leq \frac{|\eta(\zeta, \varrho)|}{2 \sqrt[q]{n}}\left(\frac{1}{2}\right)^{2-\frac{2}{q}}\left\{\left(\sum_{i=1}^{n} m^{i}\left|\psi^{\prime}\left(\frac{\varsigma}{m^{i}}\right)\right|^{q} k_{1}(s)+\left|\psi^{\prime}(\varrho)\right|^{q} \sum_{i=1}^{n} k_{2}(s)\right)^{\frac{1}{q}}\right. \\
& \left.+\left(\sum_{i=1}^{n} m^{i}\left|\psi^{\prime}\left(\frac{\varsigma}{m^{i}}\right)\right|^{q} k_{2}(s)+\left|\psi^{\prime}(\varrho)\right|^{q} \sum_{i=1}^{n} k_{1}(s)\right)^{\frac{1}{q}}\right\} .
\end{aligned}
$$

Furthermore, for $q=1$, Using the same procedure step by step as in Theorem 11, we are led to the required result. 
Corollary 14. If we put $n=m=1$ and $s=1$ in Theorem 15 , then

$$
\begin{aligned}
& \left|\frac{\psi(\varrho)+\psi(\varrho+\eta(\varsigma, \varrho))}{2}-\frac{1}{\eta(\zeta, \varrho)} \int_{\varrho}^{\varrho+\eta(\varsigma, \varrho)} \psi(x) d x\right| \\
& \leq \frac{|\eta(\zeta, \varrho)|}{8}\left[\left(\frac{\left|\psi^{\prime}(\varsigma)\right|^{q}}{4}+\frac{3\left|\psi^{\prime}(\varrho)\right|^{q}}{4}\right)^{\frac{1}{q}}+\left(\frac{3\left|\psi^{\prime}(\varsigma)\right|^{q}}{4}+\frac{\left|\psi^{\prime}(\varrho)\right|^{q}}{4}\right)^{\frac{1}{q}}\right] .
\end{aligned}
$$

Corollary 15. If we put $s=m=1$ and $\eta(\varsigma, \varrho)=\varsigma-\varrho$ in Theorem 15, we obtain inequality (4.5) in [27].

Corollary 16. If we put $n=m=1, s=1$, and $\eta(\varsigma, \varrho)=\varsigma-\varrho$ in Theorem 15 , then we obtain Corollary 6 in [27].

\section{Applications}

In this section, we remember the following special means of two positive real numbers.

(1) The arithmetic mean.

$$
A=A(\varsigma, \varrho)=\frac{\varsigma+\varrho}{2}, \quad \varsigma, \varrho \geq 0
$$

(2) The geometric mean.

$$
G=G(\zeta, \varrho)=\sqrt{\zeta \varrho}, \quad \varsigma, \varrho \geq 0
$$

(3) The harmonic mean.

$$
H=H(\varsigma, \varrho)=\frac{2 \varsigma \varrho}{\varsigma+\varrho}, \quad \varsigma, \varrho>0
$$

(4) The logarithmic mean.

$$
L=L(\varsigma, \varrho)=\frac{\varrho-\varsigma}{\ln \varrho-\ln \varsigma}, \quad \zeta, \varrho>0
$$

(5) The identric mean.

$$
I=I(\varsigma, \varrho)=\frac{1}{e}\left(\frac{\varrho^{\varrho}}{\varsigma^{\varsigma}}\right)^{\frac{1}{\varrho-\varsigma}}, \quad \varsigma, \varrho>0 .
$$

These means have many applications in areas and different types of numerical approximations. However, the following simple relationships are known in the literature.

$$
H(\zeta, \varrho) \leq G(\zeta, \varrho) \leq L(\zeta, \varrho) \leq A(\zeta, \varrho)
$$

Proposition 4. Let $\varsigma, \varrho \in[0, \infty)$ with $\varsigma<\varrho$ and $s \in[0,1]$; then,

$$
\frac{n}{2 \sum_{i=1}^{n}\left(1-\frac{s}{2}\right)^{i}} A(\zeta, \varrho) \leq L(\varsigma, \varrho) \leq A(\varsigma, \varrho) \frac{1}{n} \sum_{i=1}^{n}(2-s)^{i}
$$

Proof. If we put $\psi(x)=x$ in the above Remark 6, then we obtain the following above Inequality (10).

Proposition 5. Let $\varsigma, \varrho \in[0, \infty)$ with $\varsigma<\varrho$ and $s \in[0,1]$; then,

$$
\frac{n}{2 \sum_{i=1}^{n}\left(1-\frac{s}{2}\right)^{i}} A^{2}(\zeta, \varrho) \leq L_{2}^{2}(\varsigma, \varrho) \leq A\left(s^{2}, \varrho^{2}\right) \frac{1}{n} \sum_{i=1}^{n}(2-s)^{i}
$$

Proof. If we put $\psi(x)=x^{2}$ in the above Remark 6 , then we obtain the following above Inequality (11). 
Proposition 6. Let $\varsigma, \varrho \in[0, \infty)$ with $\varsigma<\varrho$ and $s \in[0,1]$; then,

$$
\frac{n}{2 \sum_{i=1}^{n}\left(1-\frac{s}{2}\right)^{i}} A^{n}(\varsigma, \varrho) \leq L_{n}^{n}(\zeta, \varrho) \leq A\left(\varsigma^{n}, \varrho^{n}\right) \frac{1}{n} \sum_{i=1}^{n}(2-s)^{i}
$$

Proof. If we put $\psi(x)=x^{n}$ in the above Remark 6, then we obtain the following above Inequality (12).

Proposition 7. Let $\zeta, \varrho \in(0, \infty)$ with $\varsigma<\varrho$ and $s \in[0,1]$; then,

$$
\frac{n}{2 \sum_{i=1}^{n}\left(1-\frac{s}{2}\right)^{i}} A^{-1}(\zeta, \varrho) \leq L^{-1}(\varsigma, \varrho) \leq H^{-1}(\varsigma, \varrho) \frac{1}{n} \sum_{i=1}^{n}(2-s)^{i}
$$

Proof. If we put $\psi(x)=x^{-1}, x \in(0, \infty)$ in the above Remark 6 , then we obtain the following above Inequality (13).

Proposition 8. Let $\varsigma, \varrho \in(0, \infty)$ with $\varsigma<\varrho$ and $s \in[0,1]$; then,

$$
\frac{n}{2 \sum_{i=1}^{n}\left(1-\frac{s}{2}\right)^{i}} \ln G \leq \ln I \leq \frac{\ln A}{n} \sum_{i=1}^{n}(2-s)^{i}
$$

Proof. If we put $\psi(x)=-\ln x, x \in(0,1]$ in the above Remark 6 , then we obtain the following above Inequality (14).

Remark 7. The above-discussed means, namely arithmetic, geometric, harmonic, and logarithmic, are well known in the literature because these means have remarkable applications in machine learning, probability, statistics, and numerical approximation.

\section{Conclusions}

In this article, first, we introduced a new notion of the preinvex function, i.e., the generalized $s$-type $m$-preinvex function, and elaborated some useful properties regarding this function. Hermite-Hadamard-type inequalities for the new notion are deduced. In addition, we presented some new integral inequalities involving the generalized stype $m$-preinvex function. Many existing results in the literature become the particular cases for these results as mentioned in the remarks. In the future, we hope the results of this paper and the new idea can be extended in different directions such as fractional calculus, quantum calculus, time scale calculus, etc. We hope the consequences and techniques of this article will energize and inspire researchers to explore more interesting avenues in this area.

Author Contributions: Conceptualization, M.T. and S.K.S.; methodology, M.T., A.A.S., S.K.S. and H.A.; software, M.T., A.A.S., S.K.S. and H.A.; validation, H.A., T.S. and J.R.; formal analysis, A.A.S., H.A., T.S. and J.R.; investigation, M.T., S.K.S. and H.A.; resources, M.T., A.A.S., S.K.S., H.A., T.S. and J.R.; writing—original draft preparation, M.T. and S.K.S.; writing-review and editing, M.T., S.K.S. and H.A., T.S.; visualization, T.S. and J.R.; supervision, A.A.S., H.A., T.S. and J.R.; project administration, M.T., A.A.S., S.K.S., H.A., T.S. and J.R.; funding acquisition, H.A., T.S. and J.R. All authors have read and agreed to the published version of the manuscript.

Funding: This research was funded by King Mongkut's University of Technology North Bangkok. Contract no. KMUTNB-64-KNOW-32.

Institutional Review Board Statement: Not applicable.

Informed Consent Statement: Not applicable. 
Data Availability Statement: No data were used to support this study.

Acknowledgments: King Mongkut's University of Technology North Bangkok. Contract no. KMUTN B-64-KNOW-32.

Conflicts of Interest: The authors declare that there are no conflicts of interest regarding the publication of this paper.

\section{References}

1. Özcan, S.; İşcan, İ. Some new Hermite-Hadamard type integral inequalities for the $s$-convex functions and theirs applications. $J$. Inequal. Appl. 2019, 201, 1-14

2. Niculescu, C.P.; Persson, L.E. Convex Functions and Their Applications; Springer: New York, NY, USA, 2006.

3. Xi, B.Y.; Qi, F. Some integral inequalities of Hermite-Hadamard type for convex functions with applications to means. J. Funct. Spaces. Appl. 2012, 2012, 980438. [CrossRef]

4. Khan, M.A.; Chu, Y.M.; Khan, T.U.; Khan, J. Some new inequalities of Hermite-Hadamard type for s-convex functions with applications. Open Math. 2017, 15, 1414-1430. [CrossRef]

5. Hadamard, J. Étude sur les propriétés des fonctions entiéres en particulier d'une fonction considéréé par Riemann. J. Math. Pures. Appl. 1893, 58, 171-215.

6. Mitrinovic, D.S.; Lackovic, I.B. Hermite and convexity functions. Aequ. Math. 1985, 28, 229-232. [CrossRef]

7. Beckenbach, E.F. Convex functions. Bull. Am. Math. Soc. 1948, 54, 439-460. [CrossRef]

8. Baleanu, D.; Purohit, S.D. Chebyshev type integral inequalities involving the fractional hypergeometric operators. Abstr. Appl. Anal. 2014, 2014, 609160. [CrossRef]

9. Tariq, M.; Sahoo, S.K.; Ahmad, H.; Sitthiwirattham, T.; Soontharanon, J. Several integral inequalities of Hermite-Hadamard type related to k-fractional conformable integral operators. Symmetry 2021 13, 1880. [CrossRef]

10. Butt, S.I.; Kashuri, A.; Tariq, M.; Nasir, J.; Aslam, A.; Geo, W. Hermite-Hadamard-type inequalities via n-polynomial exponentialtype convexity and their applications. Adv. Differ. Equ. 2020, 508, 1-25. [CrossRef]

11. Guzmán, P.M.; Kórus, P.; Valdés, J.E.N. Generalized integral inequalities of Chebyshev type. Fractal Fract. 2020, 4, 10. [CrossRef]

12. İşcan, İ; Turhan, S.; Maden, S. Hermite-Hadamard and simpson-like type inequalities for differentiable $p$-quasi convex Functions. Filomat 2017, 31, 5945-5953. [CrossRef]

13. Treanta, S.; Arana-Jimenez, M. KT-pseudoinvex multidimensional control problem. Optim. Control Appl. Methods 2018, 4, 1291-1300. [CrossRef]

14. Treanta, S. Mititelu, S. Efficiency for variational control problems on Riemann manifolds with geodesic quasiinvex curvilinear integral functionals. Rev. Real Acad. Cienc. Exactas Fis. Nat. Ser. A Mat. 2020, 114, 113. [CrossRef]

15. Treanta, S. Some results on $(\rho, b, d)$-variational inequalities. J. Math. Inequl. 2020, 14, 805-818. [CrossRef]

16. Sahoo, S.K.; Tariq, M.; Ahmad, H.; Nasir, J.; Aydi, H.; Mukheimer, A. New Ostrowski-type fractional integral inequalities via generalized exponential-type convex functions and applications. Symmetry 2021, 13, 1429. [CrossRef]

17. Sahoo, S.K.; Ahmad, H.; Tariq, M.; Kodamasingh, B.; Aydi, H.; De la Sen, M. Hermite-Hadamard type inequalities involving k-fractional operator for $(\bar{h}, m)$-convex Functions. Symmetry 2021, 13, 1686. [CrossRef]

18. Ahmad, H.; Tariq, M.; Sahoo, S.K.; Baili, J.; Cesarano, C. New estimations of Hermite-Hadamard type integral inequalities for special functions. Fractal Fract. 2021 5, 144. [CrossRef]

19. Latif, M.A. Hermite-Hadamard-type inequalities for geometrically r-convex functions in terms of Stolarskys mean with applications to means. Adv. Differ. Equ. 2021, 2021, 1-25

20. Latif, M.A. New weighted Hermite-Hadamard type inequalities for differentiable-convex and quasi-convex mappings. J. Math. 2021, 2021, 4495588. [CrossRef]

21. Hanson, M.A. On sufficiency of Kuhn-Tucker conditions. J. Math. Anal. Appl. 1981, 80, 545-550. [CrossRef]

22. Weir, T.; Mond, B. Pre-invex functions in multiple objective optimization. J. Math. Anal. Appl. 1988, 136, 29-38. [CrossRef]

23. Weir, T.; Jeyakumar, V. A class of nonconvex functions and mathematical programming. Bull. Aust. Math. Soc. 1988, 38, 177-189. [CrossRef]

24. Toader, G. Some generalizations of the convexity. In Proceedings of the Colloquium on Approximation and Optimization (Cluj-Napoca, 1985); University of Cluj-Napoca: Cluj-Napoca, Romania, 1985; pp. 329-338.

25. Latif, M.A.; Shoaib, M. Hermite-Hadamard type integral inequalities for differentiable $m$-preinvex and $(\alpha, m)$-preinvex functions. J. Egypt. Math. Soc. 2015, 23, 236-241. [CrossRef]

26. Deng, Y.; Kalsoom, Y.; Wu, S. Some new Quantum Hermite-Hadamard-type estimates within a class of generalized $(s, m)-$ preinvex functions. Symmetry 2019, 11, 1283.

27. Toplu, T.; Kadakal, M.; İşcan, İ. On n-polynomial convexity and some relatd inequalities. AIMS Maths 2020, 5, $1304-1318$.

28. Du,T.T.; Liao, J.G.; Li, Y.J. Properties and integral inequalities of Hadamard-Simpson type for the generalized ( $s, m)-$ preinvex functions. J. Nonlinear Sci. Appl. 2016, 9, 3112-3126. [CrossRef]

29. Noor, M.A. Hadamard integral inequalities for product of two preinvex function. Nonl. Anal. Forum. 2009, 14, 167-173.

30. Noor, M.A. Hermite-Hadamard integral inequalities for log-preinvex functions. J. Math. Anal. Approx. Theory 2007, 2, 126-131. 
31. Barani, A.; Ghazanfari, A.G.; Dragomir, S.S. Hermite-Hadamard inequality for functions whose derivatives absolute values are preinvex. J. Ineq. Appl. 2012, 2012, 1-9. [CrossRef]

32. Noor, M.A. On Hadamard integral inequalities invoving two log-preinvex functions. J. Inequal. Pure Appl. Math. 2007, 8, 1-6.

33. Antczak, T. On G-preinvex functions in mathematical programming. J. Comput. Appl. Math. 2008, 217, 212-226. [CrossRef]

34. Antczak, T. r-preinvexity and r-invexity in mathematical programming. J. Comput. Appl. Math. 2005, 250, 551-566. [CrossRef]

35. Yang, X.M.; Li, D. On properties of preinvex functions. J. Math. Anal. Appl. 2001, 256, 229-241. [CrossRef]

36. Awan, M.U.; Talib, S.; Noor, M.A.; Chu, Y.M.; Noor, K.I. Some trapezium-like inequalities involving functions having strongly n-polynomial preinvexity property of higher order. J. Funct. Spaces 2020, 2020, 9154139. [CrossRef]

37. Du, T.S.; Liao, J.G.; Chen, L.Z.; Awan, M.U. Properties and Riemann-Liouville fractional Hermite-Hadamard inequalities for the generalized $(\alpha, m)$-preinvex functions. J. Inequal. Appl. 2016, 2016, 1-24. [CrossRef]

38. Kashuri, A.; Latif, M.A. Some new parameterized inequalities for preinvex functions via generalized fractional integral operator and their applications. J. Frac. Calc. Appl. 2021, 12, 46-68.

39. Mehmood, S.; Zafar, F.; Latif, M.A.; Yasmin, N. Fejér-Hermite-Hadamard inequalities for $n$-times differentiable preinvex functions. Tbil. Math. J. 2021, 14, 255-270.

40. Mohan, S.R.; Neogy, S.K. On invex sets and preinvex functions. J. Math. Anal. Appl. 1995, 189, 901-908. [CrossRef]

41. Rashid, S.; İşcan, İ; Baleanu, D.; Chu, D.Y.M. Generation of new fractional inequalities via n-polynomials s-type convexity with applications. Adv. Differ. Equ. 2020, 2020, 1-20. [CrossRef]

42. Mishra, S.K.; Giorgi, G. Invexity and Optimization; Springer Science \& Business Media: Berlin/Heidelberg, Germany, 2008; Volume 88. 